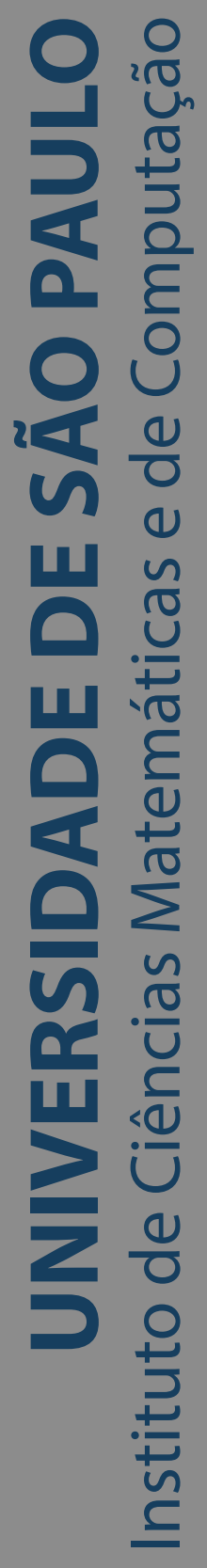

\title{
Precificação de opções exóticas utilizando CUDA
}

\section{Felipe Boteon Calderaro}

Dissertação de Mestrado do Programa de Mestrado Profissional em Matemática, Estatística e Computação Aplicadas à Indústria (MECAI) 

Assinatura:

\title{
Felipe Boteon Calderaro
}

\section{Precificação de opções exóticas utilizando CUDA}

\begin{abstract}
Dissertação apresentada ao Instituto de Ciências Matemáticas e de Computação - ICMC-USP, como parte dos requisitos para obtenção do título de Mestre - Mestrado Profissional em Matemática, Estatística e Computação Aplicadas à Indústria. VERSÃO REVISADA
\end{abstract}

Área de Concentração: Matemática, Estatística e Computação

Orientador: Prof. Dr. Adenilso da Silva Simão 
Ficha catalográfica elaborada pela Biblioteca Prof. Achille Bassi e Seção Técnica de Informática, ICMC/USP, com os dados fornecidos pelo(a) autor(a)

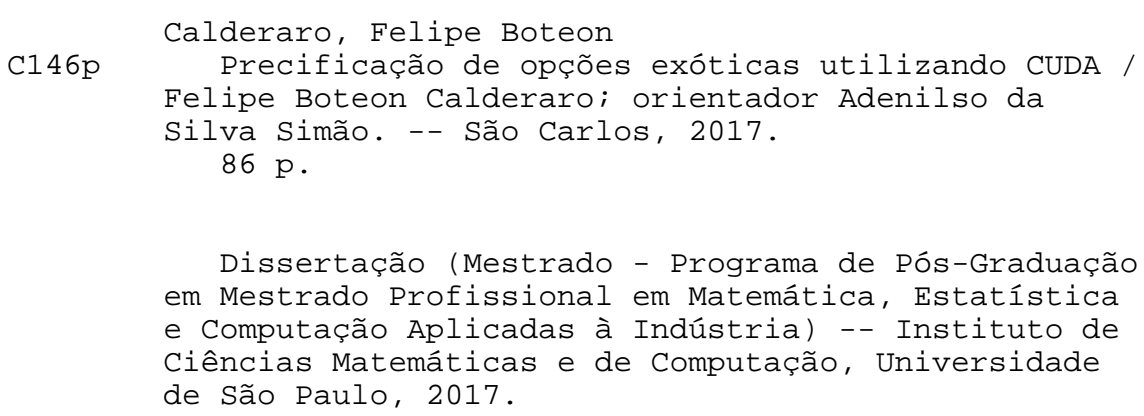

1. Precificação de derivativos. 2. Opções exóticas. 3. Geração de números aleatórios. 4. Simulação Monte Carlo. 5. CUDA. I. Simão, Adenilso da Silva, orient. II. Título. 


\title{
Felipe Boteon Calderaro
}

\section{Exotic options pricing using CUDA}

\author{
Master dissertation submitted to the Institute of \\ Mathematics and Computer Sciences - ICMC-USP, in \\ partial fulfillment of the requirements for the degree of \\ the Master - Professional Masters in Mathematics, \\ Statistics and Computing Applied to Industry. FINAL \\ VERSION \\ Concentration Area: Mathematics, Statistics and \\ Computing
}

Advisor: Prof. Dr. Adenilso da Silva Simão 

Dedico este trabalho aos meus pais que, desde sempre, acreditaram no meu potencial e me deram plenas condições para conquistar tudo que almejei até hoje. 

Agradeço aos professores Marcos Carreira, Richard Brostowicz, Adenilso Simão, Antônio Castelo Filho e todo o restante do corpo docente do programa MECAI, que contribuíram com seus valiosos conhecimentos para a realização deste trabalho.

Agradeço aos meus colegas de curso pelo companheirismo, pelas ricas discussões e pelas experiências compartilhadas.

Agradeço à minha noiva Fernanda e aos meus amigos pela paciência e compreensão nos momentos mais difíceis durante este período.

Finalmente, agradecimentos especiais são direcionados à Dorival Leão, que idealizou uma aproximação diferente da Universidade de São Paulo com o mercado de trabalho e acreditou no potencial da turma, tornando assim possível a realização do programa MECAI com ênfase em derivativos. 

"If events are random, we are not in control, and if we are in control of events, they are not random. There is therefore a fundamental clash between our need to feel we are in control and our ability to recognize randomness." (Leonard Mlodinow - The Drunkard's Walk) 



\section{RESUMO}

CALDERARO, F. B. Precificação de opções exóticas utilizando CUDA. 2017. 86 p. Dissertação (Mestrado - Mestrado Profissional em Matemática, Estatística e Computação Aplicadas à Indústria) - Instituto de Ciências Matemáticas e de Computação, Universidade de São Paulo, São Carlos - SP, 2017.

No mercado financeiro, a precificação de contratos complexos muitas vezes apoia-se em técnicas de simulação numérica. Estes métodos de precificação geralmente apresentam baixo desempenho devido ao grande custo computacional envolvido, o que dificulta a análise e a tomada de decisão por parte do trader. O objetivo deste trabalho é apresentar uma ferramenta de alto desempenho para a precificação de instrumentos financeiros baseados em simulações numéricas. A proposta é construir uma calculadora eficiente para a precificação de opções multivariadas baseada no método de Monte Carlo, utilizando a plataforma CUDA de programação paralela. Serão apresentados os conceitos matemáticos que embasam a precificação risco-neutra, tanto no contexto univariado quanto no multivariado. Após isso entraremos em detalhes sobre a implementação da simulação Monte Carlo e a arquitetura envolvida na plataforma CUDA. No final, apresentaremos os resultados obtidos comparando o tempo de execução dos algoritmos.

Palavras-chave: Precificação de derivativos, Opções exóticas, Geração de números aleatórios, Simulação Monte Carlo, CUDA. 



\section{ABSTRACT}

CALDERARO, F. B. Exotic options pricing using CUDA. 2017. 86 p. Dissertação (Mestrado Mestrado Profissional em Matemática, Estatística e Computação Aplicadas à Indústria) - Instituto de Ciências Matemáticas e de Computação, Universidade de São Paulo, São Carlos - SP, 2017.

In the financial market, the pricing of complex contracts often relies on numerical simulation techniques. These pricing methods generally present poor performance due to the large computational cost involved, which makes it difficult for the trader to analyze and make decisions. The objective of this work is to present a high performance tool for the pricing of financial instruments based on numerical simulations. The proposal is to present an efficient calculator for the pricing of multivariate options based on the Monte Carlo method, using the parallel programming CUDA platform. The mathematical concepts underlying risk-neutral pricing, both in the univariate and in the multivariate context, will be presented. After this we will detail the implementation of the Monte Carlo simulation and the architecture involved in the CUDA platform. At the end, we will present the results obtained comparing the execution time of the algorithms.

Keywords: Derivatives pricing, Exotic options, Random number generation, Monte Carlo Simulation, CUDA. 



\section{LISTA DE ILUSTRAÇÕES}

Figura 1 - Baixa dispersão . . . . . . . . . . . . . . . 46

Figura 2 - Alta dispersão . . . . . . . . . . . . . . . . . . 46

Figura 3 - Representação da Simulação Monte Carlo para o preço de um ativo ao longo do tempo . . . . . . . . . . . . . . . . . . . . . . 48

Figura 4 - Exemplo de sequência pseudo-aleatória bidimensional . . . . . . . . . . 50

Figura 5 - Exemplo de sequência quasi-aleatória bidimensional . . . . . . . . . . . 50

Figura 6 - Comparativo do poder de processamento das CPU's e GPU's em GIGA FLOPS 53

Figura 7 - Ilustração de um SM na arquitetura Kepler $(\mathrm{SMX}) \ldots \ldots$. . . . . . . . . 54

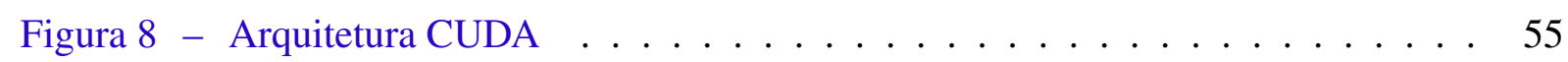

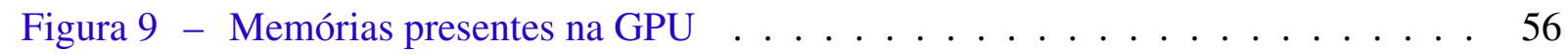

Figura 10 - Comparação de desempenho entre os generatos cuRAND em número de geração de amostras por segundo . . . . . . . . . . . . . . . 58

Figura 11 - Especificações Nvidia GeForce GTX 750 Ti - Fabricante . . . . . . . . . . 59

Figura 12 - Especificações Nvidia GeForce GTX 750 Ti - deviceQuery . . . . . . . . . 60

Figura 13 - Paralelização para a geração dos números aleatórios na GPU. . . . . . . . . 61

Figura 14 - Paralelização para a construção dos random walks correlacionados na GPU -

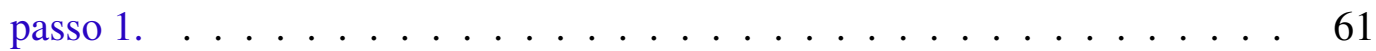

Figura 15 - Paralelização para a construção dos random walks correlacionados na GPU passo 2. . . . . . . . . . . . . . . . . . . 6 62

Figura 16 - Paralelização para a construção dos random walks correlacionados na GPU passo P. . . . . . . . . . . . . . . . . . . . 62

Figura 17 - Tempo de execução das simulações executadas. . . . . . . . . . . . . . 65 



\section{LISTA DE CÓDIGOS-FONTE}

Código-fonte 1 - Código fonte CUDA . . . . . . . . . . . . . . . . 73

Código-fonte 2 - Código fonte Sequencial . . . . . . . . . . . . . . 81 



\section{LISTA DE TABELAS}

Tabela 1 - Tempo de execução dos algoritmos (segundos) para diferentes cenários de número de threads . . . . . . . . . . . . . . . . . . . . 63

Tabela 2 - Tempo de execução dos algoritmos (segundos) para diferentes cenários de número de simulações . . . . . . . . . . . . . . . . . . . . . . . . . . . . . 64 



\title{
LISTA DE ABREVIATURAS E SIGLAS
}

\author{
CPU Central Processing Unit \\ CUDA Compute Unified Device Architecture \\ EDE Equação Diferencial Estocástica \\ FLOPS FLoating-point Operations Per Second \\ GIGA $\quad 10^{9}$ \\ GPU Graphics Processing Unit \\ GPUPU General-Purpose computing on Graphics Processing Unit \\ RAM Random Access Memory \\ SM Streaming Multiprocessor
}





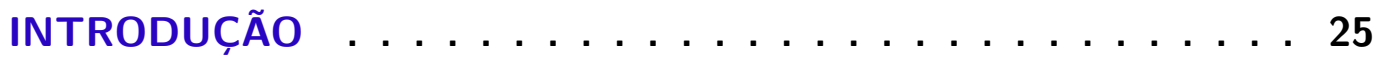

2.1 Movimento Browniano . . . . . . . . . . . . . . . . 29

2.1.1 Padrão: Processo de Wiener . . . . . . . . . . . . . . . . . 29

2.1.2 Movimento Browniano com drift . . . . . . . . . . . . . . . . . . 30

2.1.3 Movimento Browniano Geométrico . . . . . . . . . . . . . . . . . . 31

2.1.4 Propriedades Básicas do Movimento Browniano . . . . . . . . . . . . 31

2.2 Movimento Browniano Geométrico Multivariado . . . . . . . . . 32

$2.3 \quad$ Cálculo de ltô $\ldots \ldots \ldots \ldots \ldots$

2.3.1 Martingale ..................... 32

2.3.2 Integral de ltô . . . . . . . . . . . . . . . . . 33

2.3.3 Propriedades da Integral de Itô . . . . . . . . . . . . . . . . 34

2.3.4 Lema de ttô . . . . . . . . . . . . . . . . . . . . . 34

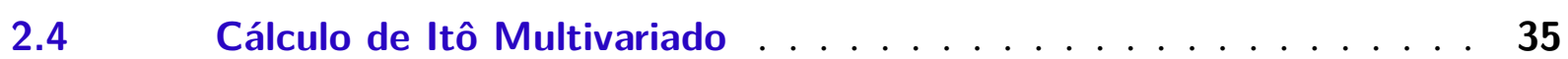

2.4.1 Integral de ltô . . . . . . . . . . . . . . . . . . . 35

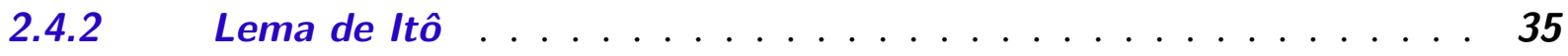

$2.5 \quad$ Black \& Scholes . . . . . . . . . . . . . . . . . 36

2.5.1 O modelo de Black \& Scholes Multivariado . . . . . . . . . . . . 37

$2.6 \quad$ Considerações Finais . . . . . . . . . . . . . . . . . 38

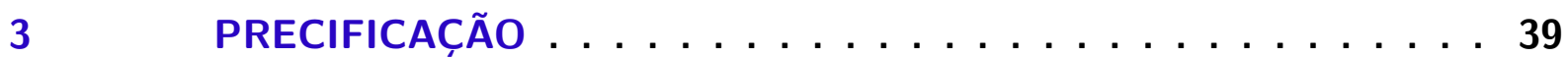

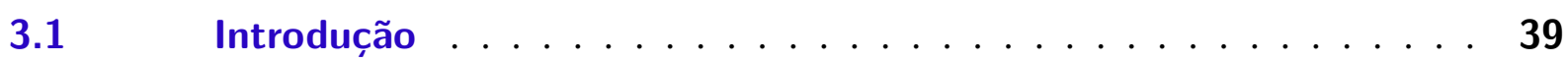

$3.2 \quad$ Estratégias e Arbitragem . . . . . . . . . . . . . . . 39

$3.3 \quad$ Medidas Equivalentes . . . . . . . . . . . . . . . . 40

3.3.1 Derivada de Radon-Nikodym . . . . . . . . . . . . . . . . 40

$3.4 \quad$ Precificação . . . . . . . . . . . . . . . . . 41

3.4.1 Teorema de Girsanov . . . . . . . . . . . . . . . . . . 43 
$3.5 \quad$ Considerações Finais . . . . . . . . . . . . . . . 43

4 SIMULAÇÃO . . . . . . . . . . . . . . . 45

$4.1 \quad$ Opções multidimensionais . . . . . . . . . . . . . 45

4.1.1 Rainbow Options . . . . . . . . . . . . . . . . . . . . . 45

4.1.1.1 Worst-of Option . . . . . . . . . . . . . . . . . . 46

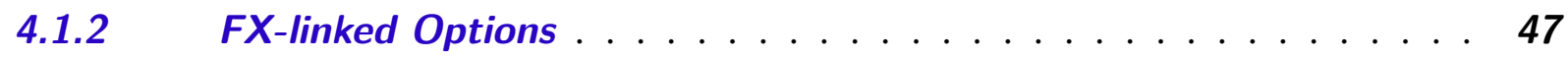

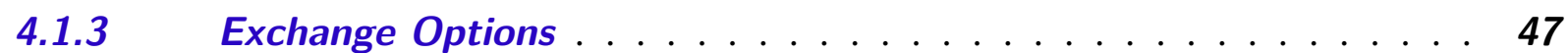

$4.2 \quad$ Monte Carlo . . . . . . . . . . . . . . . . . . . . 47

4.2 .1 Método .................... 48

4.3 Geração de Números Aleatórios . . . . . . . . . . . . . . . . . 49

$4.4 \quad$ Considerações Finais . . . . . . . . . . . . . . . . 50

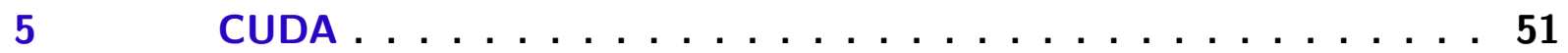

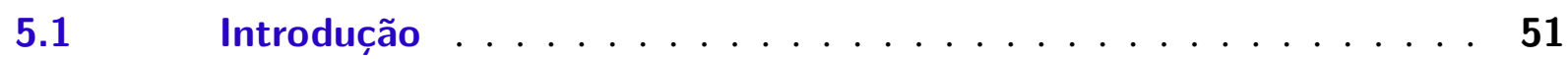

$5.2 \quad$ Arquitetura e desempenho . . . . . . . . . . . . 52

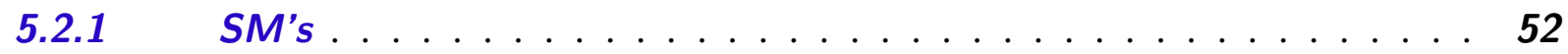

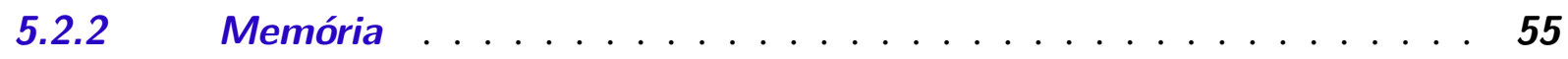

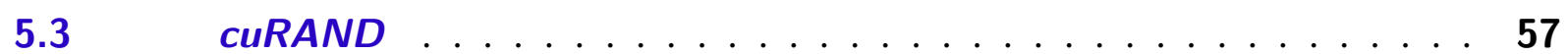

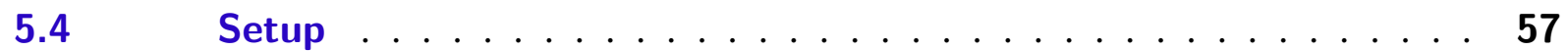

$5.5 \quad$ Algoritmo . . . . . . . . . . . . . . . 58

$5.6 \quad$ Considerações Finais . . . . . . . . . . . . . . 62

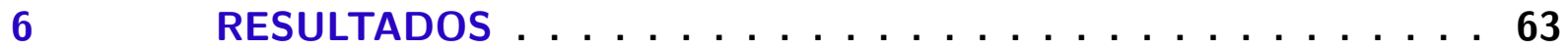

$6.1 \quad$ Threads por bloco . . . . . . . . . . . . . 63

6.2 Desempenho do Algoritmo . . . . . . . . . . . . 64

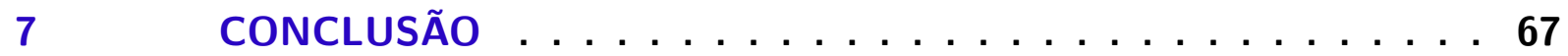

REFERÊNCIAS . . . . . . . . . . . . . . . . . 69

APÊNDICE A DECOMPOSIÇÃO DE CHOLESKY . . . . . . . 71

APÊNDICE B CÓDIGO-FONTE CUDA . . . . . . . . 73

APÊNDICE C CÓDIGO-FONTE SEQUENCIAL . . . . . . . 81 


\section{1}

INTRODUÇÃO

\subsection{Contexto}

Time is money. Esta máxima foi empregada para descrever o que hoje chamamos de custo de oportunidade por Benjamin Franklin em 1748, em um ensaio intitulado Advice to a Young Tradesman (FRANKLIN, 1748). No contexto da negociação eletrônica, esta afirmação não apenas se mantém como pode ser interpretada em seu sentido mais literal.

O mercado financeiro vem passando por uma revolução em termos de tecnologia. O trading eletrônico popularizou-se e é um caminho sem volta. A cada dia o algorithmic trading ganha mais força, e a cada trade os algoritmos ficam mais complexos. Interpretação automática de notícias para a tomada de decisão e aprendizado de máquina já são realidade nos mercados mais desenvolvidos.

Milhões de ordens de compra e venda automatizadas são executadas a cada pregão em intervalos de milésimos de segundo e, neste mercado, uma estratégia de negociação eficiente é garantia de retorno financeiro. Desta forma, o desempenho é ponto crucial no contexto deste trabalho. Com players cada vez mais automatizados, algoritmos de negociação eletrônica e ferramentas de análise mais apuradas, a tempestividade na precificação é fator decisivo em cada negócio, principalmente quando falamos sobre derivativos.

\subsection{Motivação / Problema}

O mercado de derivativos é composto majoritariamente por contratos de futuros, swaps e opções, subdividindo-se em várias modalidades. Neste trabalho, nosso foco recai sobre uma destas subdivisões, as opções exóticas, dado o modelo de apreçamento normalmente praticado.

Opções exóticas são estruturas que geralmente possuem soluções analíticas bastante complexas ou, em alguns casos, esta solução simplesmente não existe. Desta forma, os preços 
destes produtos são usualmente calculados através de simulações numéricas, processos trabalhosos que exigem uma grande carga de processamento computacional. Opções com barreira, autocalls e basket options são exemplos clássicos, amplamente abordados na literatura. Apesar da complexidade, alguns tipos destas opções são bastante negociados no mercado, como por exemplo as opções com barreira.

Indo além da camada de negociação, este problema também aparece no contexto do gerenciamento de risco, onde as rotinas para a marcação a mercado dos produtos em instituições com carteiras muito grandes demandam horas de processamento. Desta forma, as opções exóticas representam um ótimo grupo de estudo para este trabalho.

\subsection{Proposta}

A proposta deste estudo é a construção de uma calculadora eficiente e acessível para a precificação de derivativos exóticos, em particular, opções multidimensionais, ou seja, opções sobre um conjunto de ativos. Utilizamos, para isto, a Simulação Monte Carlo implementada na plataforma CUDA de programação paralela. Esta escolha deve-se basicamente a uma combinação de dois fatores: desempenho e custo. Os benchmarks das gerações mais recentes das placas gráficas Nvidia demonstram que a performance da arquitetura paralela pode ser muito bem empregada por um preço razoavelmente acessível, principalmente se comparado a grandes estruturas de processamento, como clusters especializados.

\subsection{Síntese dos Resultados}

A técnica de paralelização apresentou bons resultados e mostrou-se uma ótima alternativa para a implementação da calculadora, dados o custo da solução e o esforço envolvidos. Enquanto a solução sequencial apresentou tempos de execução crescentes de forma linear e proporcionais ao número de simulações executadas, a solução utilizando GPU manteve-se relativamente constante, apresentando tempos de execução consideravelmente menores que o algoritmo sequencial em alguns casos. Todas as simulações executadas via GPU apresentaram tempos de execução menores que 1 segundo.

\subsection{Organização do Trabalho}

Este texto está organizado da seguinte maneira: no primeiro capítulo serão relembrados os conceitos básicos do cálculo estocástico, como o Movimento Browniano, Martingales e o Cálculo de Itô. Além disto, será revisto o modelo clássico de precificação de opções desenvolvido por Fischer Black e Myron Scholes em 1973. Estes serão os pilares para o desenvolvimento do restante do trabalho. 
A seguir, no capítulo Precificação, serão abordados os conceitos de medidas equivalentes e será demonstrada a não-arbitragem no processo de precificação por simulação de preços.

No capítulo seguinte, Simulação, serão apresentados o conceito da Simulação Monte Carlo, uma explicação objetiva do método e as particularidades desta implementação. Também fazem parte do escopo deste capítulo a geração de números aleatórios e as opções multidimensionais, além de exemplos de produtos negociados.

No capítulo CUDA, serão abordados os detalhes em torno da plataforma: histórico, arquiteturas, ferramentas, gerenciamento de memória e bibliotecas específicas. Será apresentado o setup utilizado para a execução do algoritmo, bem como uma breve explicação sobre o racional utilizado na codificação.

Finalmente, serão exibidos os resultados das simulações através da comparação do tempo de execução dos algoritmos paralelo e sequencial e, em seguida, serão apresentadas as considerações finais. 



\section{CONCEITOS BÁSICOS}

Antes de discutir a precificação dos produtos em si, vamos recapitular alguns conceitos importantes para este trabalho. Serão apresentados tópicos como o Processo de Wiener, o Movimento Browniano Geométrico (univariado e multivariado), dentre outros necessários para a construção de uma simulação consistente. Além disso, relembraremos o Modelo de Black \& Scholes para que possamos validar os preços obtidos através desta simulação, bem como os principais assuntos que embasam a precificação dos derivativos no contexto de risco neutro.

\subsection{Movimento Browniano}

\subsubsection{Padrão: Processo de Wiener}

O processo de Wiener é um fenômeno observado em diversas áreas do conhecimento, como por exemplo na física, onde representa a descrição do movimento aleatório das partículas suspensas nos fluidos. Nomeado em homenagem a Norbert Wiener, também é frequentemente observado no mundo da matemática aplicada, economia e nas finanças quantitativas, contexto principal deste estudo. É frequentemente chamado de movimento browniano padrão, em homenagem ao botânico Robert Brown, que em 1827 observou partículas presas em cavidades dentro de grãos de pólen na água (BROWN, 1828).

Este processo é de extrema importância para este trabalho, pois representa a base da representação do comportamento aleatório dos preços dos ativos ao longo da linha do tempo. Vamos às condições básicas: no mundo das finanças, um processo estocástico $\{W(t): t \geq 0\}$ é considerado um movimento browniano padrão com $x \in R$ se:

- $W(0)=x$

- O processo tem incrementos independentes, ou seja, para todos os tempos $0 \leq t_{1} \leq$ $t_{2} \leq \ldots \leq t_{n}$ os incrementos $W\left(t_{n}\right)-W\left(t_{n-1}\right), W\left(t_{n-1}\right)-W\left(t_{n-2}\right), \ldots, W\left(t_{2}\right)-W\left(t_{1}\right)$ são 
variáveis aleatórias independentes.

- Para todo $t \geq 0$ e $h>0$, os incrementos $W(t+h)-W(t)$ são normalmente distribuídos com média zero e desvio padrão $h$.

- Quase certamente ${ }^{1}$, a função $t \mapsto W(t)$ é contínua.

Para cada $t>0$ a variável aleatória $W(t)=W(t)-W(0)$ é o incremento em $[0, t]$ : é normalmente distribuída com média zero, variância $t$ e função de densidade de probabilidade

$$
f_{W_{t}}(x)=\frac{1}{\sqrt{2 \pi t}} e^{-x^{2} /(2 t)}
$$

Para $p \in[0,1]$ o $p$-ésimo percentil de $W(t)$ é $N^{-1}(p) \sqrt{t}$, onde $N^{-1}$ é a função inversa da distribuição normal

$$
N(x)=\frac{1}{\sqrt{2 \pi}} \int_{-\infty}^{x} e^{-u^{2} / 2} d u
$$

\subsubsection{Movimento Browniano com drift}

O Movimento Browniano com drift $X(t)$ é a solução da EDE com drift constante e coeficientes de difusão

$$
d X(t)=\mu d t+\sigma d W(t)
$$

com valor inicial $X(0)=x_{0}$. Integrando temos

$$
X(t)=x_{0}+\mu t+\sigma W(t)
$$

Admitindo que $X(t)$ é normalmente distribuído, com média $x_{0}$ e variância $\sigma^{2} t$, sua função densidade é

$$
f_{W_{t}}(x)=\frac{1}{\sigma \sqrt{2 \pi t}} e^{-\left(x-x_{0}-\mu t\right)^{2} / 2 \sigma^{2} t}
$$

Para $p \in[0,1]$ o $p$-ésimo percentil é $x_{0}+\mu t+N^{-1}(p) \sqrt{t}$.

1 Seja $(\Omega, \mathscr{F}, \mathbb{P})$ um espaço de probabilidade. Um evento $E \in \mathscr{F}$ acontece quase certamente se $P[E]=1$. De maneira equivalente, $E$ acontece quase certamente se a probabilidade de $E$ não acontecer é zero: $P\left[E^{C}\right]=0$. De maneira mais geral, qualquer evento $E$ (não necessariamente em $\mathscr{F}$ acontece quase certamente se $E^{C}$ está contido num conjunto nulo: um subconjunto de $N \in \mathscr{F}$ tal que $P[N]=0$ (JACOD; PROTTER, 2004)). 


\subsubsection{Movimento Browniano Geométrico}

O Movimento Browniano Geométrico $X(t)$ é a solução da EDE com drift linear e coeficientes de difusão

$$
d X(t)=\mu X(t) d t+\sigma X(t) d W(t)
$$

com valor inicial $X(0)=x_{0}$.

$\mathrm{Na}$ equação, o termo $\mu X(t) d t$ representa a tendência da trajetória (drift), enquanto o termo $\sigma X(t) d W(t)$ é responsável pelo efeito de ruído aleatório. Uma aplicação simples do Lema de Itô (a ser abordado com mais detalhes a seguir), $\operatorname{com} F(X)=\log (X)$ nos leva à solução

$$
X(t)=x_{0} e^{\hat{\mu} t}+\sigma W(t)
$$

onde $\hat{\mu}=\mu-\frac{1}{2} \sigma^{2}$.

Uma vez que $X(t)$ é normalmente distribuído, temos:

- Média: $E(X(t))=x_{0} e^{\mu t}$

- Variância: $\operatorname{var}(X(t))=x_{0}^{2} e^{2 \mu t}\left(e^{\sigma^{2} t}-1\right)$

- Densidade: $f_{W_{t}}(x)=\frac{1}{\sigma x \sqrt{2 \pi t}} e^{-\left(\log x-\log x_{0}-\hat{\mu} t\right)^{2} / 2 \sigma^{2} t}$

Para $p \in[0,1]$ the $p$-ésimo percentil é $x_{0} e^{\hat{\mu} t+N^{-1}(p) \sigma \sqrt{t}}$

\subsubsection{Propriedades Básicas do Movimento Browniano}

É importante citar algumas propriedades importantes do Movimento Browniano, como a propriedade de Markov e a propriedade forte de Markov.

- Propriedade de Markov: Seja $X(t)$ um processo estocástico num espaço de probabilidade $(\Omega, \mathscr{F}, \mathbb{P}), \operatorname{com} X(t)$ adaptado à filtragem $\mathscr{F} . X(t)$ obedece à propriedade de Markov se, para cada tempo $s$ e $t \geq 0$,

$$
\mathbb{P}\left(X(s+t) \in \delta_{x} \mid F_{s}\right)=\mathbb{P}\left(X(s+t) \in \delta_{x} \mid X(s)\right)
$$

- Propriedade Forte de Markov: Seja $X(t)$ um processo estocástico num espaço de probabilidade $(\Omega, \mathscr{F}, \mathbb{P}), \operatorname{com} X(t)$ adaptado à filtragem $\mathscr{F} . X(t)$ obedece à propriedade de Markov se, para cada tempo finito de parada $\tau$ e $t \geq 0$,

$$
\mathbb{P}\left(X(\tau+t) \in \delta_{x} \mid F_{\tau}\right)=\mathbb{P}\left(X(\tau+t) \in \delta_{x} \mid X(\tau)\right)
$$


onde $\mathscr{F}_{\tau}=\left[\mathscr{M} \in \mathscr{F} \mid \mathscr{M} \cap(\tau \leq t) \in \mathscr{F}_{t} \forall t \geq 0\right]$.

De maneira mais direta, a propriedade de Markov nos diz que, a qualquer ponto $s$ fixado no tempo, o futuro da trajetória de um movimento browniano é dependente somente do estado presente do processo, e não requer nenhum conhecimento adicional sobre a história do mesmo. A propriedade forte de Markov nos diz que isto é verdade não apenas para qualquer ponto determinístico no tempo $s$, mas também para qualquer tempo de parada $\tau$ (MÖRTERS; PERES, 2010).

\subsection{Movimento Browniano Geométrico Multivariado}

Na seção anterior, definimos as características do movimento browniano univariado. A generalização deste modelo para múltiplas dimensões leva em conta a correlação entre os diversos movimentos brownianos. Vamos considerar $X(t)=\left(X_{1}(t), \ldots, X_{d}(t)\right)^{\top}$ como sendo um processo estocástico multivariado definido como

$$
d X_{i}(t)=X_{i}(t) \mu_{i} d t+X_{i}(t) \sigma_{i} d W_{i}(t)
$$

com $W$ sendo um vetor de processos de Wiener correlacionados de maneira que $\mathbb{E}\left[d W_{i} d W_{j}\right]=$ $\rho_{i j} d t$ para $i \neq j$, onde $\rho_{i j}$ representa a covariância entre os processos de Wiener $i$ e $j ; E\left[d W_{i}^{2}\right]=$ $d t$; e $d t^{2}=0$. Desta maneira, $X$ é chamado de movimento browniano geométrico multivariado.

\subsection{Cálculo de Itô}

O cálculo de Itô, nomeado em homenagem a Kiyoshi Itô, amplia os conceitos do cálculo tradicional para o cálculo estocástico, e é peça fundamental para a construção deste trabalho. O conceito central do cálculo de Itô é a integral de Itô, a ser abordada no próximo tópico. Referências completas com demonstrações e provas dos conceitos a serem apresentados podem ser encontradas em (SHREVE; KARATZAS, 1991) e (ØKSENDAL, 2003).

\subsubsection{Martingale}

Seja $X(t)$ um processo estocástico com $E^{P}(|X(t)|)<\infty \forall t \in[0, T]$. Dizemos que $X(t)$ é um martingale na medida de probabilidade $P$ se, para todo $s, t \in[0, T] \operatorname{com} t \leq s$ :

$$
E_{t}^{P}(X(s))=X(t)
$$

De maneira direta, um martingale é um processo estocástico tal que o valor esperado de uma observação em um tempo $t$, dadas todas as observações até algum tempo anterior $s$, é igual a observação no tempo anterior $s$. 
Se substituirmos o sinal de igualdade em 2.11 por $\leq$ ou $\geq, X(t)$ é classificado como supermartingale e submartingale, respectivamente.

\subsubsection{Integral de Itô}

Conforme citado anteriormente, a integral de Itô é uma extensão da integral de Riemann para o contexto do cálculo estocástico, onde trabalhamos com um integrador não diferenciável.

O teorema fundamental do cálculo nos permite calcular as integrais de Riemann sem retornar à sua definição original. No cálculo de Itô, este papel é desempenhado pelo lema de Itô, a ser abordado na sequência. O lema de Itô possui em sua expressão um termo adicional, que aparece devido à não-suavidade dos caminhos descritos pelo movimento browniano.

Seja $\mathscr{F}$ a filtragem gerada por um movimento browniano até o tempo $t$, e seja $F(t) \in \mathscr{F}$ um processo estocástico adaptado. De maneira similar à aproximação de somas para a integral de Riemann, definiremos a seguinte aproximação para a integral de Itô:

$$
Y_{\Delta t}(t)=\sum_{t_{k}<t} F\left(t_{k}\right) \Delta W_{k}
$$

com as notações $t_{k}=k \Delta t$, e $\Delta W_{k}=W\left(t_{k+1}\right)-W\left(t_{k}\right)$. Se o limite existe, a integral de Itô é

$$
Y(t)=\lim _{\Delta t \rightarrow \infty} Y_{\Delta t}(t)
$$

Há alguma flexibilidade nesta definição, mas ainda bem menor que na integral de Riemann. É essencial que utilizemos diferenças futuras ao invés de diferenças passadas (onde teríamos $\left.\Delta W_{k}=W\left(t_{k}\right)-W\left(t_{k-1}\right)\right)$, pois então

$$
\mathbb{E}\left[F\left(t_{k}\right) \Delta W_{k} \mid \mathscr{F}_{t_{k}}\right]=0
$$

Cada um dos termos na somatória em 2.12 são $\mathscr{F}$-mensuráveis, $\log _{0} Y_{n}(t)$ também é. Se verificarmos em tempos discretos $t_{n}, Y_{\Delta t}$ é um martingale:

$$
\mathbb{E}\left[Y_{\Delta t}\left(t_{n+1}\right) \mid \mathscr{F}\right]=Y \Delta t\left(t_{n}\right)
$$

No limite $\Delta t \rightarrow 0$ faz com que $Y(t)$ também seja um martingale $\mathscr{F}$-mensurável.

O exemplo mais famoso é:

$$
Y(T)=\int_{0}^{T} W(t) d W(t)=\frac{1}{2}\left(W(t)^{2}-T\right)
$$




\subsubsection{Propriedades da Integral de Itô}

$$
I(t)=\int_{0}^{t} \delta(u) d B(u)
$$

Suas principais propriedades são:

- Adaptabilidade: Para cada $t, I(t)$ é $\mathscr{F}$-mensurável.

- Linearidade: Se

$$
I(t)=\int_{0}^{t} \delta(u) d B(u), \quad J(t)=\int_{0}^{t} \gamma(u) d B(u)
$$

então

$$
I(t) \pm J(t)=\int_{0}^{t}(\delta(u) \pm \gamma(u)) d B(u)
$$

e

$$
c I(t)=\int_{0}^{t} c \delta(u) d B(u)
$$

- Martingale: $\mathrm{I}(\mathrm{t})$ é um martingale.

- Continuidade: I(t) é uma função contínua do limite de integração $t$.

- Isometria de Itô: $\mathbb{E}\left(I^{2}(t)\right)=\mathbb{E}\left(\int_{0}^{t} \delta^{2}(u) d u\right)$

\subsubsection{Lema de Itô}

O Lema de Itô é um dos resultados mais importantes do cálculo de Itô. Representa uma extensão da regra da cadeia para a integral de Itô, e é usado na matemática para encontrar a diferencial de uma função de um tipo particular de processo estocástico. Nas finanças, sua aplicação mais comum é na demonstração da fórmula de Black \& Scholes. Conforme visto na seção sobre o Movimento Browniano Geométrico, o preço de um ativo pode ser descrito pela seguinte equação:

$$
d X=\mu(X, t) d t+\sigma(X, t) d W
$$

onde $W$ é um processo de Wiener e $\mu$ e $\sigma$ são funções de $X$ e $t$. Seja $F: \mathbb{R}$ x $\mathbb{R}^{+} \rightarrow \mathbb{R},(x, t) \mapsto$ $F(x, t)$ uma função contínua e duplamente diferenciável. Então $F$ obedece ao processo

$$
d F=\left(\frac{\partial F}{\partial X} \mu+\frac{\partial F}{\partial t}+\frac{1}{2} \frac{\partial^{2} F}{\partial X^{2}} \sigma^{2}\right) d t+\frac{\partial F}{\partial X} \sigma d W
$$




\subsection{Cálculo de Itô Multivariado}

\subsubsection{Integral de Itô}

Vamos assumir de maneira geral que o vetor de preços $X(t)$ é descrito por um vetor de processos de Itô

$$
X(t)=X(0)+\int_{0}^{t} \mu(s, \omega) d s+\int_{0}^{t} \sigma(s, \omega) d W(s)
$$

Escrevendo na notação diferencial:

$$
d X(t)=\mu(t, \omega) d t+\sigma(t, \omega) d W(t)
$$

É possível encontrar uma descrição detalhada da integral de Itô multivariada em (SHREVE; KARATZAS, 1991). Para nosso propósito, vamos considerar a integral de Itô como

$$
\int_{o}^{t} \sigma(s, \omega) d W(s)=\lim _{n \rightarrow \infty} \sum_{i=1}^{n} \sigma((i-1) \delta, \omega)[W(i \delta)-W((i-1) \delta)]
$$

onde $\delta \triangleq t / n$. É importante notar também que o integrando $\sigma$ é sempre computado à esquerda de cada intervalo $[(i-1) \delta, i \delta]$, conforme já citado no caso univariado.

As propriedades da integral de Itô se mantêm para o caso multivariado: adaptabilidade, linearidade, martingale, continuidade e isometria de Itô.

\subsubsection{Lema de Itô}

Seja $f(t, x), x=\left(x_{1}, \cdots, x_{p}\right)^{\top}$ uma função contínua, $f:[0, T] \times \mathbb{R}^{p} \rightarrow \mathbb{R}$, com derivadas parciais contínuas $\partial f / \partial t=f_{t}, \partial f / \partial x_{i}=f_{x}, \partial^{2} f / \partial x_{i} \partial x_{j}=f_{x_{i} x_{j}}$. Seja $X(t)$ descrito pelo processo de Itô (2.24) e $Y(t)=f(t, X(t))$ um processo escalar. Desta maneira, $Y(t)$ é um proceso de Itô com equação diferencial

$$
\begin{aligned}
d Y(t)= & f_{t}(t, X(t)) d t+f_{x}(t, X(t)) \mu(t, \omega) d t \\
& +f_{x}(t, X(t)) \sigma(t, \omega) d W(t) \\
& +\frac{1}{2} \sum_{i=1}^{p} \sum_{j=1}^{p} f_{x_{i}} f_{x_{j}}(t, X(t))\left(\sigma(t, \omega) \sigma(t, \omega)^{\top}\right)_{i, j} d t
\end{aligned}
$$

Para o caso onde $p=d=1$ obtemos a expressão apresentada no capítulo anterior. 


\subsection{Black \& Scholes}

Em 1973, Fischer Black e Myron Scholes utilizaram o modelo de mercado financeiro introduzido por Samuelson para precificar opções, obedecendo a determinadas condições (BLACK; SCHOLES, 1973). Esta teoria foi um marco na história da precificação dos derivativos, sendo utilizada amplamente no mercado financeiro até hoje. Neste trabalho, vamos nos apoiar neste modelo para a validação da simulação construída, através da comparação do preço simulado com o preço teórico, calculado pelo modelo de Black \& Scholes para opções simples univariadas (plain vanilla).

Para a teoria de Black \& Scholes as seguintes condições são estabelecidas:

1. O preço do ativo $S$ obedece à equação do Movimento Browniano Geométrico

$$
d S_{t}=S_{t} \mu d t+S_{t} \sigma d W_{t}
$$

com $W_{t}$ sendo um processo de Wiener e $\mu$ e $\sigma$ constantes.

2. Comprar e vender ativos é permitido para qualquer quantidade finita da mesma.

3. Não há custos ou taxas de transação.

4. Não há pagamento de dividendos durante a existência do derivativo.

5. Não há oportunidades de arbitragem.

6. A compra e venda de ativos acontece de maneira contínua no tempo.

7. A taxa de juros livre de risco ( $r$ ) é constante e igual para quaisquer vencimentos.

Considere um mercado que obedece às condições descritas anteriormente com uma opção $V\left(S_{t}, T\right)$, ou seja, com ativo subjacente $S_{t}$ e data de vencimento $T$. Utilizando o Lema de Itô temos:

$$
d V_{t}=\frac{\partial V}{\partial t} d t+\frac{\partial V}{\partial S} d S_{t}+\frac{1}{2} \frac{\partial^{2} V}{\partial S^{2}} d S_{t}^{2}
$$

onde $d S_{t}^{2}$ representa a variação quadrática de $S_{t}$. Sabemos de 2.27 que $d S_{t}^{2}=\sigma^{2} S_{t}^{2} d t$, e conseguentemente, no tempo $t$ temos:

$$
d V_{t}=\left[\frac{\partial V}{\partial t}+\frac{1}{2} \sigma^{2} S^{2} \frac{\partial^{2} V}{\partial S^{2}}\right] d t+\frac{\partial V}{\partial S} d S_{t}
$$

O termo dentro dos parênteses é todo determinístico, e o termo que aparece junto a $d S_{t}$ é o único termo estocástico. Podemos remover o termo estocástico com a construção de um 
portifólio $\Pi_{t}$, comprado em uma opção (com valor $V_{t}$ ) e vendido em $\partial V / \partial S$ unidades do ativo subjacente

$$
\Pi_{t}=V_{t}-\frac{\partial V}{\partial S} S_{t}
$$

Isto representa a seguinte equação diferencial estocástica:

$$
d \Pi_{t}=d V_{t}+\frac{\partial V}{\partial S} d S_{t}
$$

e de 2.29 temos:

$$
d \Pi_{t}=\left[\frac{\partial V}{\partial t}+\frac{1}{2} \sigma^{2} S^{2} \frac{\partial^{2} V}{\partial S^{2}}\right] d t
$$

Como o crescimento de $\Pi_{t}$ ao longo do tempo é livre de risco, podemos igualá-lo ao título livre de risco $B_{t}^{d}$, que é descrito por:

$$
d B_{t}^{d}=r^{d} B_{t}^{d} d t
$$

Logo:

$$
d \Pi_{t}=r^{d} \Pi_{t} d t
$$

onde $r^{d}$ representa a taxa de juros doméstica. Igualando os termos em 2.32 e 2.34 temos:

$$
\begin{aligned}
\frac{\partial V}{\partial t}+\frac{1}{2} \sigma^{2} S^{2} \frac{\partial^{2} V}{\partial S^{2}} & =r^{d} \Pi_{t} \\
& =r^{d}\left[V_{t}-\frac{\partial V}{\partial S} S\right]
\end{aligned}
$$

o que nos leva à famosa equação de Black \& Scholes:

$$
\frac{\partial V}{\partial t}+\frac{1}{2} \sigma^{2} S^{2} \frac{\partial^{2} V}{\partial S^{2}}+r^{d} S \frac{\partial V}{\partial S}-r^{d} V_{t}=0
$$

É importante notar neste ponto que o drift $(\mu)$ não aparece na equação.

\subsubsection{O modelo de Black \& Scholes Multivariado}

Nesta seção, ampliaremos o conceito do modelo de Black \& Scholes, conforme demonstrado em 1985 em (COX; JR; ROSS, 1985).

Seja $n \in \mathbb{N}$ e considerando um mercado consistido de um ativo livre de risco $B$ e $k$ ativos $X_{i=1}^{k}$ sujeitos a risco. Estabelecemos então, condições análogas às vistas anteriormente: 
1. O preço do vetor de ativos $X$ obedece a equação do movimento browniano geométrico multivariado

$$
d X_{i}(t)=X_{i}(t) \mu_{i} d t+X_{i}(t) \sigma_{i} d W_{i}(t)
$$

com $W_{i}(t)$ sendo um processo de Wiener e $\mu$ e $\sigma$ constantes.

As demais condições sobre compra e venda de ativos, custos de transação, dividendos, taxa de juros e arbitragem se mantêm as mesmas do cenário univariado (condições 2 - 7).

Este é o chamado modelo generalizado de Black \& Scholes (ESPOSITO, 2010). Considerando uma opção européia $F$ com data de vencimento $T$, a equação (generalizada) de Black \& Scholes é descrita como

$$
r F=\frac{\partial F}{\partial t}+r Z^{\top} \frac{\partial F}{\partial X}+\frac{1}{2} \operatorname{tr}\left(\sigma^{\top} D H D \sigma\right)
$$

onde $\operatorname{tr}(\cdot)$ é o traço da matriz $k \times k$. O traço é a somatória dos elementos na diagonal principal da matriz. $H$ é a matriz Hessiana de $F$ e $D$ é dado por

$$
D=\left(\begin{array}{cccc}
X_{1} & 0 & \cdots & 0 \\
0 & X_{2} & \cdots & 0 \\
\vdots & \vdots & \cdot & \vdots \\
0 & 0 & \cdots & X_{4}
\end{array}\right)
$$

\subsection{Considerações Finais}

Neste capítulo foram apresentados os conceitos do Movimento Browniano, do Cálculo de Itô e da teoria de Black \& Scholes. Esta é a base para a construção das variáveis aleatórias e precificação de ativos multivariados ao longo do tempo. No próximo capítulo abordaremos o apreçamento de ativos e a construção de portifólios de maneira mais generalizada. 
CAPÍTULO

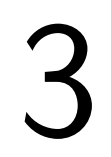

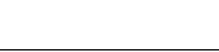

PRECIFICAÇÃO

\subsection{Introdução}

Neste capítulo serão discutidos os conceitos de arbitragem e medidas equivalentes, que representam condições primordiais para a construção da Simulação Monte Carlo, a ser apresentada no capítulo seguinte.

\subsection{Estratégias e Arbitragem}

Considere um investidor utilizando uma estratégia envolvendo $p$ ativos $X_{1}, \cdots, X_{p}$. Esta estratégia é caracterizada pelo processo $\phi(t)=\left(\phi_{1}(t), \cdots, \phi_{p}(t)\right)^{\top}$, com $\phi_{i}(t)$ sendo a quantidade comprada do ativo $i$ no tempo $t$. Deste modo, o valor $\pi(t)$ da estratégia no tempo $t$ é

$$
\pi(t)=\phi(t)^{\top} X(t)
$$

O ganho realizado entre uma pequena quantidade de tempo $[t, t+\delta]$ é aproximadamente $\phi(t)^{\top} X[(t+\delta)-X(t)]$, o que nos sugere que a integral de Itô é um bom modelo para descrever ganhos sobre $[0, T]$.

Uma estratégia é dita auto-financiável se as mudanças no portifólio são causadas apenas por perdas ou ganhos, sem adição ou subtração de fundos. De maneira mais formal, a estratégia é dita auto-financiável se, para qualquer $t \in[0, T]$

$$
\pi(t)-\pi(0)=\int_{0}^{t} \phi(s)^{\top} d X(s)
$$


Definido $\pi(t)$ em 3.1, chamamos de arbitragem uma estratégia $\phi$ para a qual $\pi(0)=0$ e, para algum $t \in[0, T]$,

$$
\pi(t) \geq 0 \quad \text { e } P(\pi(t)>0)>0
$$

A não existência da arbitragem é fundamental para a consistência dos processos que descrevem os preços dos ativos (BJÖRK, 2009).

\subsection{Medidas Equivalentes}

Precisamos inicialmente descrever as condições nas quais garantimos a inexistência de oportunidades de arbitragem, e para tal abordaremos as medidas martingale equivalentes e teoremas fundamentais, como a derivada de Radon-Nikodym.

\subsubsection{Derivada de Radon-Nikodym}

Considere duas medidas de probabilidade $P$ e $\hat{P}$ no mesmo espaço amostral $(\Omega, \mathscr{F})$. Estas medidas são ditas equivalentes se $P(A)=0 \Leftrightarrow \hat{P}=0, \forall A \in \mathscr{F}$, ou seja, as duas medidas possuem os mesmos subconjuntos nulos.

Admitindo a condição anterior (medidas equivalentes), o teorema de Radon-Nikodym diz que existe uma única variável aleatória não-negativa $R \operatorname{com} \mathbb{E}^{P}(R)=1$ tal que

$$
\hat{P}(A)=\int_{A} R d P
$$

A variável $R$ é chamada derivada de Radon-Nikodym, e é denotada por $d \hat{P} / d P$. Associaremos esta variável com o processo de densidade

$$
\zeta(t)=\mathbb{E}_{t}^{P}\left(\frac{d \hat{P}}{d P}\right), \quad \forall t \in[0, T]
$$

onde $\zeta(t)$ é um $P$-martingale com $\zeta(0)=1$ e $\zeta(t)=\mathbb{E}_{t}^{P}(\zeta(T))$ (MUSIELA; RUTKOWSKI, 2006). Demonstramos então que, para qualquer variável aleatória $\mathscr{F}_{T}$-mensurável $Y(T)$, com $R=d \hat{P} / d P$,

$$
\begin{aligned}
\mathbb{E}^{P}\left(Y(T) \mid \mathscr{F}_{t}\right) & =\frac{1}{\mathbb{E}^{P}\left(R \mid \mathscr{F}_{t}\right)} \mathbb{E}^{P}\left(R Y(T) \mid \mathscr{F}_{t}\right) \\
& =\zeta(t)^{-1} \mathbb{E}^{P}\left(\mathbb{E}^{P}\left(R \mid \mathscr{F}_{T}\right) Y(T) \mid \mathscr{F}_{t}\right) \\
& =\mathbb{E}^{P}\left(Y(T) \frac{\zeta(T)}{\zeta(t)} \mid \mathscr{F}_{t}\right)
\end{aligned}
$$


Vamos também definir um elemento importante para a normalização dos preços dos ativos, o deflator. Chamaremos o deflator de $D(t)$ e definiremos o processo normalizado $\left.X^{D}(t)=\left(X_{1}(t) / D(t), \cdots, X_{p}(t) / D(t)\right)\right)^{\top}$. Dizemos que a medida $Q^{D}$ é uma medida martingale equivalente induzida por $D$ se $X^{D}(t)$ for um martingale com respeito a $Q^{D}$. Se $Q^{D}$ é uma medida martingale, dizemos que uma estratégia auto-financiável é possível se

$$
\int_{0}^{t} \phi(s)^{\top} d X^{D}(s)
$$

também for um martingale. A combinação do lema de Itô com as equações 3.1 e 3.2 implica que $\pi(t) / D(t)$ é um $Q^{D}$-martingale quando a estratégia é possível.

Para as estratégias possíveis, a existência de medidas martingales equivalentes é de extrema importância pois se existe um deflator $D$ de maneira que a série de preços normalizada permita uma medida martingale equivalente, então não há arbitragem.

Finalmente, se o deflator é um dos $p$ ativos, o deflator é chamado numeraire. Digamos que $X_{1}$ é estritamente positivo e pode ser usado como um numeraire e que $D(t)$ é um deflator conforme descrito previamente. Como $X_{1}(t) / D(t)$ é um $Q^{D}$-martingale, podemos usar o teorema de Radon-Nikodym para definir uma nova medida $Q^{X_{1}}$ utilizando a função densidade $\zeta(t)=$ $\left(X_{1}(t) / D(t)\right) /\left(X_{1}(0) / D(0)\right)$. Temos então, para uma variável $Y(T) \mathscr{F}_{T}$-mensurável

$$
X_{1}(t) \mathbb{E}_{t}^{Q^{X_{1}}}\left(\frac{Y(T)}{X_{1}(T)}\right)=D(t) E_{t}^{Q^{D}}\left(\frac{Y(T)}{D(T)}\right)
$$

\subsection{Precificação}

Imagine um derivativo com vencimento $T$ que pague uma variável aleatória $V(T) \mathscr{F}_{T}$ mensurável, e não faça nenhum outro pagamento antes de $T$. Assumimos que $V(T)$ possui variância finita e que este derivativo exista se houver uma estratégia $\phi$ de tal forma que $V(T)=$ $\phi(T)^{\top}, X(T)=\pi(T)$. Dizemos que esta estratégia replica o derivativo. Importante: a ausência de arbitragem garante que no tempo 0 o preço de um derivativo $V(T)$ precisa ser igual ao custo de entrada na estratégia auto-financiável, ou seja, $V(0)=\pi(0)$. E de maneira generalizada, $V(t)=\pi(t), t \in[0, T]$.

Esta observação nos permite precificar derivativos como valores esperados sob uma determinada medida martingale equivalente. De maneira mais específica, considere um deflator $D$ e assuma a existência de uma medida martingale equivalente $Q^{D}$ induzida por $D$. Conforme abordado previamente, a condição de não-arbitragem é garantida pela existência de $Q^{D}(\mathrm{AN}$ DERSEN; PITERBARG, 2010). Agora, da propriedade de martingale de $\pi(t) / D(t)$ na medida 
$Q^{D}$ e da relação $V(t)=\pi(t)$ segue que

$$
\frac{V(t)}{D(t)}=\mathbb{E}_{t}^{Q^{D}}\left(\frac{V(T)}{D(T)}\right)
$$

Ou seja

$$
V(t)=D(t) \mathbb{E}_{t}^{Q^{D}}\left(\frac{V(T)}{D(T)}\right)
$$

Se todas as variáveis aleatórias de variância finita $\mathscr{F}$-mensuráveis podem ser replicadas, dizemos que temos um mercado completo. Num mercado completo todos os derivativos tem preço único, mas na ausência de arbitragem um mercado é completo só e somente só existir um único deflator induzindo uma única medida martingale equivalente. De 3.10 concluímos que as medidas martingales induzidas por numeraires precisam ser únicas também.

Considerando então dois numeraires $N(t)$ e $M(t)$, induzindo medidas martingales equivalentes $Q^{N}$ e $Q^{M}$, respectivamente. Se o mercado é completo então a função densidade da derivada de Radon-Nikodym relacionando duas medidas é dada unicamente por

$$
\zeta(t)=E_{t}^{Q^{N}}\left(\frac{d Q^{M}}{d Q^{N}}\right)=\frac{M(t) / M(0)}{N(t) / N(0)}
$$

Neste ponto, levaremos em conta as condições sobre os preços dos ativos que permitem a existência de uma medida martingale equivalente e os efeitos na dinâmica do ativo decorrentes da mudança de medida de probabilidade. Consideraremos duas medidas $P$ e $P(\theta)$ relacionadas por uma densidade $\zeta^{\theta}(t)=\mathbb{E}_{t}^{P}(d P(\theta) / d P)$, onde $\zeta^{\theta}(t)$ é um martingale exponencial dado pelo processo de Itô

$$
d \zeta^{\theta}(t) / \zeta^{\theta}(t)=-\theta(t)^{\top} d W(t)
$$

com $W(t)$ um processo browniano $d$-dimensional. Aplicando o Lema de Itô podemos escrever

$$
\zeta^{\theta}(t)=\exp \left(-\int_{0}^{t} \theta(s)^{\top} d W(s)-\frac{1}{2} \int_{0}^{t} \theta(s)^{\top} \theta(s) d s\right)
$$

Vale ressaltar que para definir um martingale, é necessário obedecer a condição de Novikov:

$$
\mathbb{E}\left[\exp \left(\frac{1}{2} \int_{0}^{t} \theta(s)^{\top} \theta(s) d s\right)\right]<\infty
$$




\subsubsection{Teorema de Girsanov}

Suponha que $\zeta^{\Theta}(t)$ definido anteriormente seja um martingale. Então, para todo $\in[0, T]$

$$
W^{\theta}(t)=W(t)+\int_{0}^{t} \theta(s) d s
$$

é um movimento browniano na medida de probabilidade $P(\theta)$.

Este teorema nos permite deslocar a medida de probabilidade para transformar um processo de Itô em um processo de Itô livre de drift. Dessa maneira, o preço do ativo em $P$

$$
d X(t)=\mu(t) d t+\sigma(t) d W(t)
$$

pode ser escrito como

$$
d X(t)=(\mu(t)-\sigma(t) \theta(t)) d t+\sigma(t) d W^{\theta}(t)
$$

É importante notar que uma mudança na medida de probabilidade afeta o drift $\mu$ de um processo de Itô, mas não afeta o coeficiente de difusão $\sigma$.

\subsection{Considerações Finais}

Neste capítulo foi apresentado o conceito das medidas equivalentes e a garantia da nãoarbitragem na precificação dos ativos. Foi demonstrado que, alterando a medida de probabilidade, podemos realizar uma Simulação de preço sem drift, o que embasa nosso capítulo seguinte, no qual será abordada com mais detalhes a Simulação Monte Carlo. 

Nos capítulos anteriores foram apresentados os conceitos envolvidos e demonstrada a não existência da arbitragem para os processos de descrição dos preços dos ativos. Neste capítulo serão um pouco mais detalhados os instrumentos fincanceiros alvo do nosso estudo, as opções, bem como o método utilizado para descrever os movimentos dos preços dos ativos ao longo do tempo, a Simulação Monte Carlo.

\subsection{Opções multidimensionais}

As opções multidimensionais são uma evolução natural às tradicionais opções plain vanilla, amplamente negociadas no mercado. Vamos enumerar a seguir alguns dos tipos mais comuns destas opções.

$\mathrm{Na}$ literatura há diferentes abordagens para a precificação de ativos multivariados, desde a extensão do modelo de Black \& Scholes (CLARK, 2011), utilização do método de cópulas (HANZON; SPIEKSMA, 2013), princípios de reflexão (MUIRHEAD; BOROVKOV, 2011) e métodos numéricos (IANCU, 2004). Como o foco deste trabalho é contruir uma calculadora eficiente e de simples implementação, vamos abordar com mais detalhes a precificação da worst-of option através do método numérico da Simulação Monte Carlo.

\subsubsection{Rainbow Options}

Opção que dá ao seu comprador o direito de exercê-la sobre o melhor, o pior ou uma combinação dos retornos de dois ou mais ativos num determinado período de tempo. Estas opções normalmente são feitas usando ativos com retornos correlacionados. Há inúmeras variações das rainbow options, como por exemplo: worst-of option, best-of option, mix-max option, multi-asset option, specialty option, spread option. 


\subsubsection{Worst-of Option}

A worst-of option dá ao seu detentor o direito de exercê-la sobre o ativo que pior performou, dado um conjunto, ou cesto de ativos. Esta estrutura pode ser utilizada, por exemplo, por um investidor interessado em maximizar o retorno de um ativo específico no cesto (WORSTOF..., ). Tomemos por woc o pay-off de uma call worst-of, por exemplo:

$$
w o c=\max \left[\min \left(S_{1}(T), S_{2}(T), \ldots, S_{n}(T)\right)-K, 0\right]
$$

Onde $K$ é o strike e $S_{n}(T)$ é o preço do ativo $n$ no vencimento $T$.

Esta call worst-of tem um pay-off potencial menor que uma call plain vanilla sobre o mesmo cesto de ativos, logo, também é mais barata. Este preço menor pela estrutura é o que torna as worst-of options populares nas mesas de negociação.

No entanto, para entender esta comparação entre os preços, é necessário entender o conceito de dispersão. Utilizado no contexto de trades multidimensionais, a dispersão indica o quão "parecidos"são os ativos envolvidos. A figura a seguir mostra o efeito da dispersão nos retornos dos ativos. Quanto maior a dispersão, maior é o retorno esperado do ativo com melhor performance no cesto e menor o retorno esperado do pior ativo. De maneira similar, dispersões menores levam a relativos menores níveis de retorno do melhor ativo do cesto e maiores retornos do pior ativo do cesto.

Figura 1 - Baixa dispersão

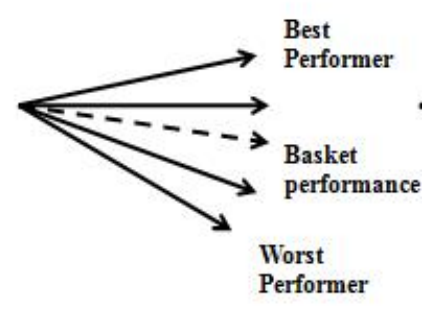

Figura 2 - Alta dispersão

Fonte: (DELTAQUANTS, 2016)

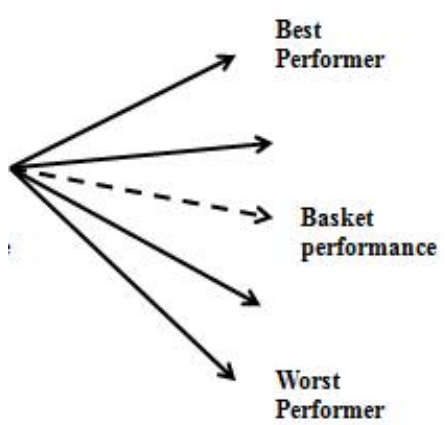

Para analisar o efeito das correlações e das volatilidades nas worst-of options, é importante entender como estes fatores afetam a dispersão. Se a correlação entre os ativos é baixa, os retornos tendem a ser bem diferentes, e vice-versa. Além disso, altos níveis de volatilidade levam a retornos com desvios consideráveis do seu valor esperado. Ainda, altos níveis de volatilidade aliados a baixos níveis de correlação entre os ativos levam a patamares ainda maiores de dispersão. 


\subsubsection{FX-linked Options}

As FX-linked Options são usualmente empregadas como instrumento de proteção para movimentos adversos em diferentes taxas de câmbio. Esta classe de opções envolve sempre um componente de taxa de câmbio cuja função é agir como um fator de conversão para mudar as unidades monetárias de uma moeda para outra. Estas opções são divididas basicamente em 3 subgrupos:

- Cross-currency options: opções sobre títulos nos quais o prêmio, o strike e o pay-off são determinados numa moeda diferente da moeda na qual os cupons e o principal dos títulos são pagos. Deste modo, o pay-off de uma cross-currency option depende tanto dos preços dos títulos quanto da taxa de câmbio.

- Quanto options: opções sobre ativos definidos numa moeda diferente da moeda doméstica do comprador. A conversão do pay-off pode ser feita com uma taxa de câmbio fixa pré-estabelecida ou com taxa de câmbio flutuante.

- Composite options: opções sobre ações estrangeiras (denominadas ou em moeda local ou em moeda estrangeira) exercidos tanto em moeda local quanto em moeda estrangeira.

\subsubsection{Exchange Options}

Opções de troca, como diz o nome. São utilizadas, por exemplo, para monetizar movimentos relativos entre ações preferenciais e ordinárias.

\subsection{Monte Carlo}

A simulação Monte Carlo é um método que se apoia na análise probabilística através da geração e amostragem de números aleatórios. Tem importantes aplicações em diversos campos da ciência, tais como física, matemática e finanças. No mundo das finanças, é uma das ferramentas mais utilizadas para se obter resultados numéricos em contextos onde a solução analítica é muito complexa.

O nome Monte Carlo vem do luxuoso distrito da ilha de Mônaco onde, assim como na modelagem econômica, a probabilidade e os resultados aleatórios são elementos centrais nos cassinos, como pode ser observado em jogos como roleta, dados e máquinas caça-níquel.

Suas principais vantagens quando aplicada à precificação de derivativos são a abrangência e a facilidade de implementação. É possível estimar pay-offs para os contratos mais complexos de maneira relativamente simples, tudo depende da abordagem probabilística com a qual é feita a simulação dos preços futuros. Suas principais desvantagens residem na generalização e no custo computacional. É grande a demanda de processamento neste método, e um dos objetivos deste trabalho é oferecer uma implementação que minimize este revés. 
A carga de processamento dá-se pela necessidade da geração de um número muito grande de simulações para garantir a margem de erro desejada, ou seja, a precisão do algoritmo. Sendo $M$ o número de simulações e $\omega$ o desvio padrão dos valores calculados pelas simulações, o erro padrão das estimativa do preço é $\omega / \sqrt{M}$.

Figura 3 - Representação da Simulação Monte Carlo para o preço de um ativo ao longo do tempo

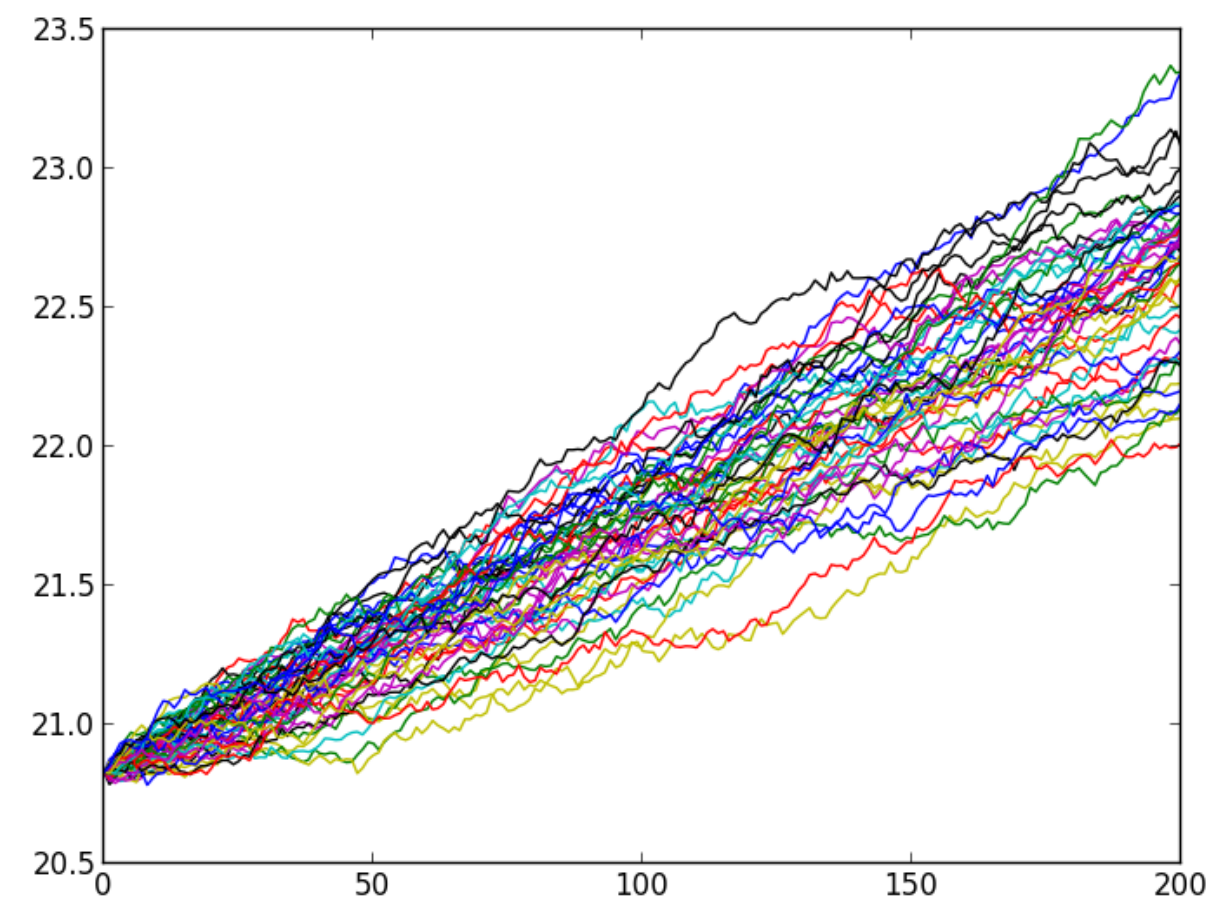

Fonte: (CONTINUUM, 2017)

\subsubsection{Método}

A simulação Monte Carlo usa como premissa a precificação risco-neutra. Neste contexto, o pay-off esperado é utilizado um procedimento de amostragem e, após isto, descontado pela taxa livre de risco. Ou seja:

1. Simulação do ativo-objeto e demais variáveis até o vencimento.

2. Cálculo dos pay-offs no vencimento.

3. Cálculo do preço da opção através da média dos pay-offs calculados trazida à valor presente pela taxa livre de risco.

É importante citar alguns detalhes e premissas da implementação realizada: 
- Como no escopo deste trabalho vamos precificar elementos multidimensionais, é importante lembrar que, para cada simulação realizada, são construídos $n$ caminhos aleatórios correlacionados, onde $n$ representa o número de ativos no cesto.

- Volatilidades, taxas de juros e matriz de correlação são constantes.

- Para fins de desempenho, a geração dos conjuntos de caminhos aleatórios correlacionados é realizada em paralelo.

\subsection{Geração de Números Aleatórios}

A geração de números aleatórios é peça fundamental em qualquer simulação, e sempre foi um grande desafio em termos computacionais. Isto se dá pelo simples fato de ser impossível produzir uma sequência genuinamente aleatória utilizando algoritmos computacionais determinísticos. Números genuinamente aleatórios são criados a partir da mensuração de eventos físicos que se acredita serem aleatórios (ruído atmosférico, por exemplo). Sequências geradas por algoritmos computacionais são chamadas pseudo-aleatórias ou quasi-aleatórias.

Uma sequência de números pseudo-aleatórios satisfaz a maior parte da propriedades estatísticas de uma sequência genuinamente aleatória, mas é gerada através de um algoritmo determinístico. Isso significa que, por maior que seja o período, sempre haverá uma repetição dos números gerados. Para se evitar tal repetição, são introduzidos nos algoritmos elementos como offsets, termo a ser abordado no capítulo seguinte.

Uma sequência de números quasi-aleatórios apresenta uma vantagem com relação à anterior: possui baixa discrepância, propriedade que mede a uniformidade na distribuição das amostras, aplicado principalmente para casos multidimensionais (notem que multidimensionais neste caso refere-se às variáveis aleatórias, e não ao contexto). Em outras palavras, nestas sequências os números são distribuídos de maneira a maximizar o espaço entre eles, prevenindo a formação de clusters, ou seja, agrupamentos de números próximos uns aos outros.

Na implementação deste trabalho, utilizamos um algoritmo pseudo-randômico. 
Figura 4 - Exemplo de sequência pseudoaleatória bidimensional

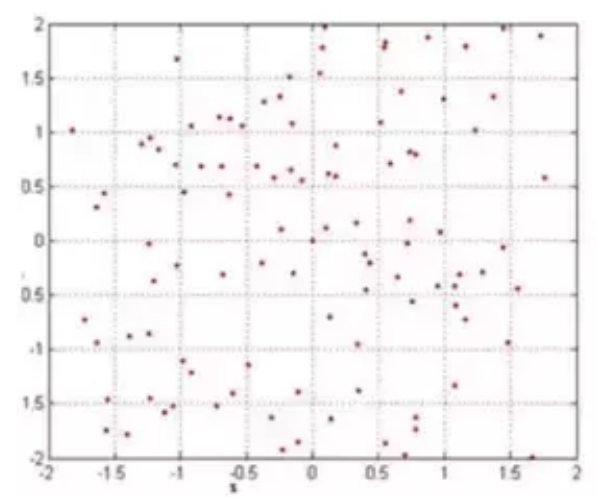

Figura 5 - Exemplo de sequência quasialeatória bidimensional

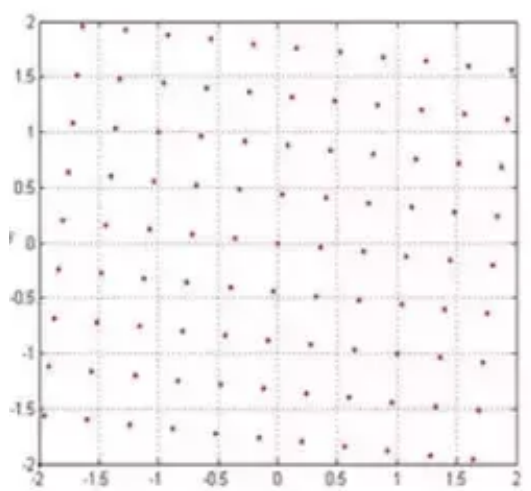

Fonte: (SENTHILNATH et al., 2013)

\subsection{Considerações Finais}

Neste capítulo foram apresentados os conceitos centrais da Simulação Monte Carlo multivariada e também algumas características e alguns exemplos reais de opções exóticas. No próximo capítulo será explorada em detalhes a ferramenta computacional utilizada para a aplicação destes conceitos e implementação da solução proposta: a plataforma CUDA de computação paralela. Serão apresentados os principais elementos da plataforma, os motivos que justificam a escolha desta ferramenta e o racional utilizado no algoritmo para a construção dos caminhos aleatórios e cálculo do preço das opções. 
CAPÍTULO

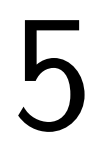

CUDA

\subsection{Introdução}

CUDA, sigla para Compute Unified Device Architecture, é uma plataforma de computação paralela desenvolvida pela Nvidia que permite aos desenvolvedores utilizarem o poder de processamento presente nas GPUs (sigla para Unidades de Processamento Gráfico, ou Graphics Processing Units em inglês) para propósitos gerais.

As GPUs são microprocessadores especializados desenvolvidos para a criação e exibição de imagens. A principal característica destes microprocessadores é a utilização da arquitetura paralela para manipulação rápida e eficiente de memória. São utilizados em sistemas embarcados, video games, computadores pessoais, smartphones e atualmente até em automóveis.

Estes microprocessadores já vem sendo discutidos e utilizados desde os primórdios do video game na década de 70, mas foi só no final da década de 90, com o lançamento da GeForce 256, que as GPU's se tornaram realmente populares. Durante este período, o hardware começou a ficar cada vez mais programável e, com a crescente (e constante) busca por desempenho, começaram a ganhar força as GPGPUs (sigla para GPUs de Propósito Geral ou General-Purpose computing on Graphics Processing Units) (NICKOLLS; DALLY, 2010). Foi então que a Nvidia, liderada por Ian Buck, começou a trabalhar no CUDA, apresentado ao mercado em 2006. Inicialmente desenvolvido apenas a linguagem $\mathrm{C}$, a plataforma hoje suporta diversas outras linguagens, como Java, Python, R, Perl, Ruby, Fortran, MATLAB, entre outras. Podem ser citados como exemplos de aplicação de CUDA:

- Renderização de gráficos 3D e manipulações de vídeos em geral;

- Criptografia e compressão aceleradas;

- Bioinformática, mapeamento de DNA; 
- Simulações para análises físicas e médicas;

- Reconhecimento facial;

- Cálculos distribuídos em geral.

A maior limitação do CUDA é que a tecnologia está disponível em alto desempenho apenas para as placas de vídeo fabricadas pela própria Nvidia. Os modelos suportados podem ser consultados diretamente no site do fabricante (NVIDIA, 2017). Os atuais toolkits publicados pela Nvidia contêm um conjunto de bibliotecas para rotinas gráficas, matemáticas e demais aplicações. Neste trabalho foi utilizada a biblioteca $c u R A N D$ para a geração de variáveis aleatórias. Abordaremos este tópico com maior nível de detalhes nas seções subsequentes.

\subsection{Arquitetura e desempenho}

O alto nível de paralelização obtido pelas placas gráficas pode ser entendido observandose a arquitetura e a estrutura de acesso à memória destes dispositivos. Tanto as GPU's quanto as CPU's atuais possuem memórias dedicadas e capacidade de execução em diferentes threads. O objetivo de cada uma das famílias de processadores, porém, é diferente: enquanto as CPU's priorizam a baixa latência na execução de comandos ordenados, a arquitetura das GPU's prioriza o throughput, ou taxa de transferência, omitindo a latência através da paralelização do processamento (KIRK; WEN-MEI, 2016). A figura 6 compara o poder computacional das GPU's e das CPU's ao longo dos anos. Pode-se notar um notável crescimento de capacidade de hardware no contexto das GPU's, mas é imprescindível o correto entendimento e aplicação da arquitetura para se conseguir de fato tirar proveito do desempenho oferecido.

As GPU's possuem dois componentes principais:

- Memória global

- Equivalente à memória RAM para a CPU

- Acessível pela GPU e pela CPU

- Streaming Multiprocessors (SM's)

- Unidades de processamento, equivalente aos cores das CPU's

- Cada SM possui unidades de controle, registradores e unidades de memória próprios, e é composto de diversos CUDA cores.

\subsubsection{SM's}

A cada nova geração das placas gráficas Nvidia, novas arquiteturas foram desenvolvidas, sempre em busca de melhor desempenho através da maximização do throughput. No caso da 
Figura 6 - Comparativo do poder de processamento das CPU's e GPU's em GIGA FLOPS

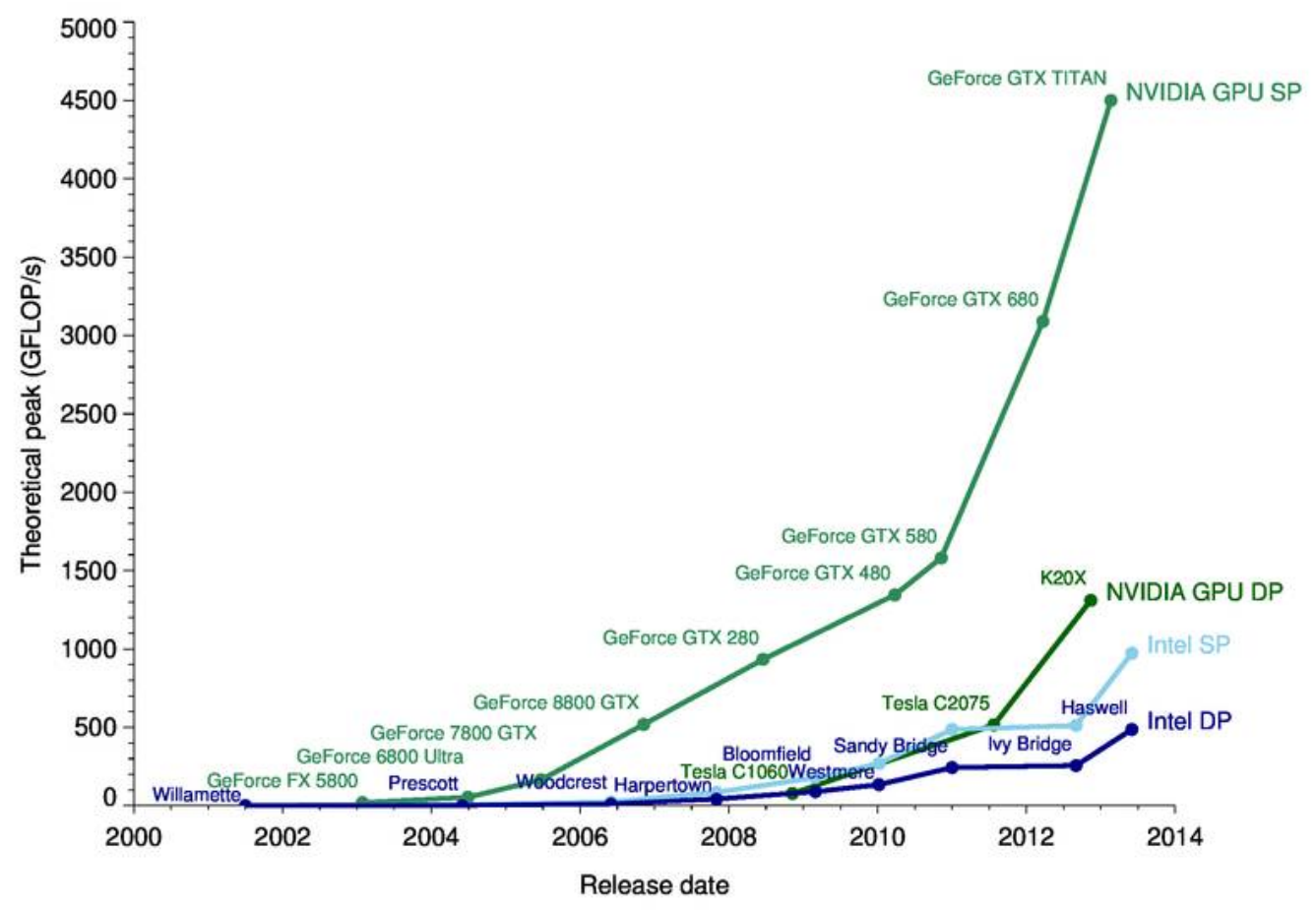

Fonte: (GALLOY, 2013)

placa gráfica utilizada neste trabalho, está presente a arquitetura Kepler (figura 7), cujos detalhes também podem ser consultados nos materiais fornecidos pelo fabricante.

Independente da geração, todas as arquiteturas possuem a mesma vantagem: conseguem executar diversas threads de maneira simultânea utilizando uma única instrução. A cada clock, todos os núcleos dentro de cada SM executam a mesma instrução ao mesmo tempo. Este grupo de threads executando o mesmo código no contexto de uma SM é chamado warp. Diversos warps dentro de um mesmo SM são gerenciados por um controlador chamado warp scheduler.

As threads são agrupadas em blocos, e este agrupamento possui um limite máximo de 1024 threads por bloco nas arquiteturas mais recentes. Cada bloco é executado pelo mesmo SM e, por esta razão, conseguem usar um mesmo bloco de memória compartilhado (a ser detalhado a seguir). Cada função CUDA é denominada kernel, e ao conjunto de blocos criados para um kernel é dado o nome de grid. O limite para o número de blocos em cada grid é extremamente grande. Na figura 8 é possível observar uma representação da arquitetura CUDA. 
Figura 7 - Ilustração de um SM na arquitetura Kepler (SMX)

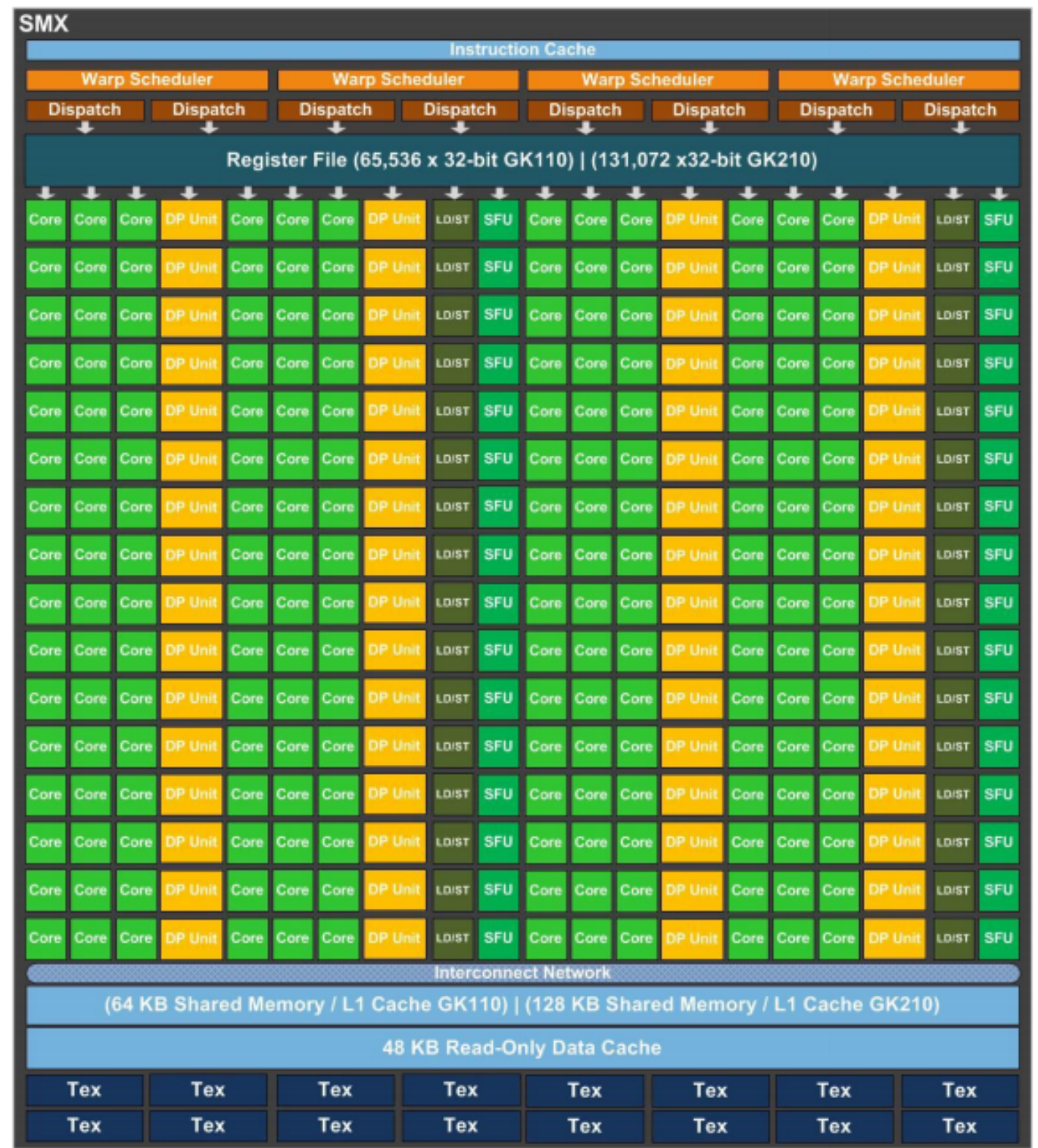

SMX: 192 single-precision CUDA cores, 64 double-precision units, 32 special function units (SFU), and 32 load/store units (LD/ST).

Fonte: (CLUA; ZAMITH, 2015)

Todas estas informações podem ser consultadas com o fabricante ou através do uso de ferramentas gratuitas, e serão exibidas adiante para a placa gráfica utilizada neste trabalho. É importante citar que, para fins de otimização, faz-se necessário checar a taxa de ocupação dos núcleos, então a distribuição de threads por bloco e de blocos por grid podem resultar em níveis diferentes de desempenho. 
Figura 8 - Arquitetura CUDA

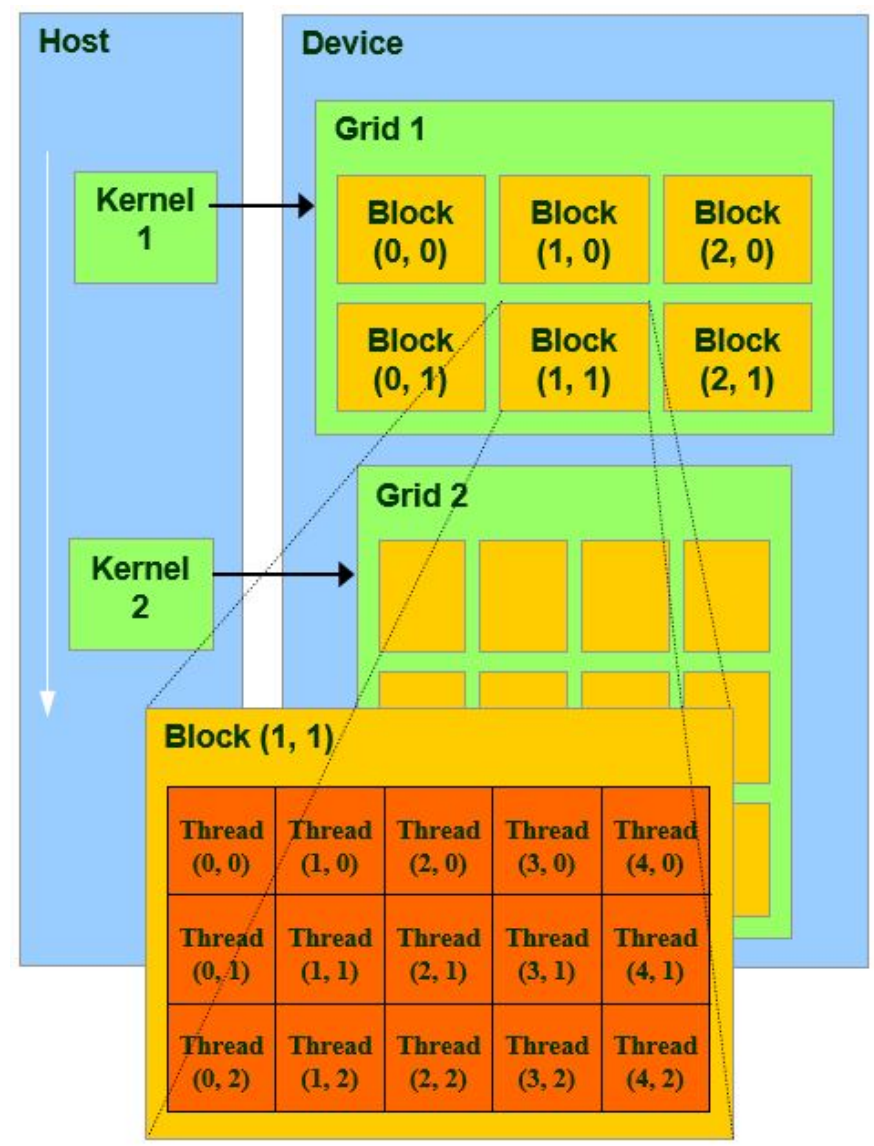

Fonte: (NVIDIA, 2017)

\subsubsection{Memória}

As GPUs possuem diferentes tipos de memória:

- Global Memory, ou Memória Global: é a memória principal da placa gráfica e a maior em tamanho (na arquitetura mais atual, Maxwell, atingem até $12 \mathrm{~Gb}$ ), contudo é a que apresenta a maior latência e o menor throughput. Por ela é feita a comunicação entre a GPU e o programa externo. Os dados são acessíveis por todos os cores e threads e persistem durante toda a existência do grid.

- Shared Memory, ou Memória Compartilhada: dedicada a cada SM, é acessível somente por threads pertencentes ao mesmo bloco. Possui baixa latência e alto throughput. Seus dados persistem durante a vida do bloco, e depois disso são apagados para que o bloco subsequente utilize o mesmo SM.

- Local Memory, ou Memória Local: é nomeada assim pois seu escopo restringe-se somente a cada thread. É a memória de mais rápido acesso, e seus dados só existem durante a 
execução da thread.

- Texture Memory: parte da memória global com desempenho diferenciado pelo fato de ser acessível através de um cache read-only. É geralmente utilizada para compartilhamento de dados entre diferentes threads.

- Constant Memory: parte pequena (geralmente 64Kb) da memória global também acessível através de um cache read-only. É utilizada para armazenar dados que não se alteram ao longo da execução do kernel.

Na figura a seguir, uma representação gráfica da disposição das diferentes memórias e canais de comunicação.

Figura 9 - Memórias presentes na GPU

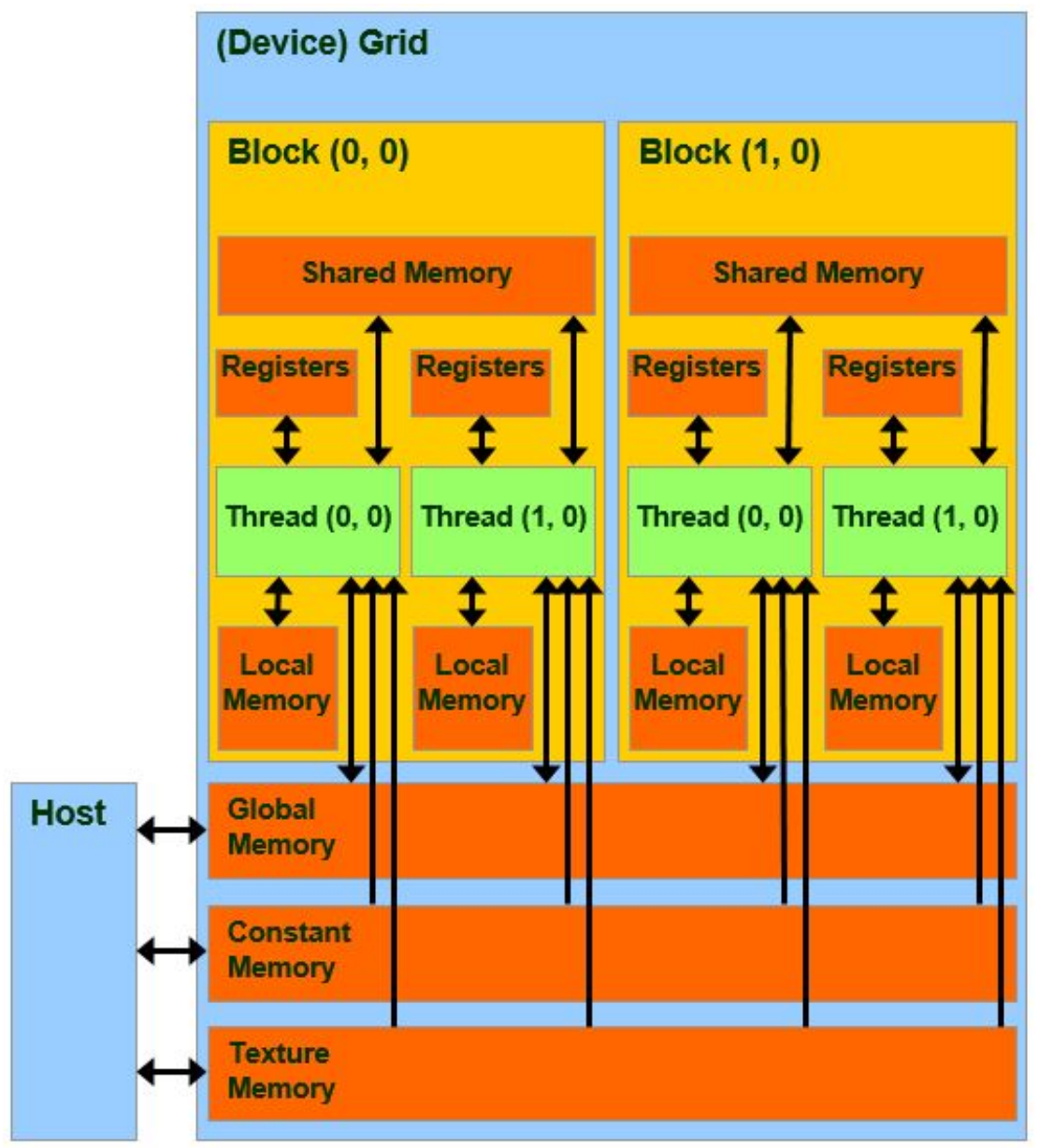

Fonte: (NVIDIA, 2017) 


\section{$5.3 \quad$ cuRAND}

Conforme citado anteriormente, um dos pontos cruciais em qualquer simulação numérica é a geração de números aleatórios. Para isto, no escopo deste trabalho utilizamos uma biblioteca específica presente no toolkit CUDA chamada cuRAND.

A cuRAND oferece um conjunto de funções que possibilita ao desenvolvedor obter uma geração simples e eficiente de números pseudo-aleatórios e quasi-aleatórios de qualidade. Há na biblioteca conjuntos diferentes de funções para execução na CPU ou na GPU.

As funções são divididas basicamente em 2 etapas: setup e geração dos números aleatórios. Durante o setup, são configurado os generators, que são os componentes responsáveis pela criação dos números aleatórios depois. Os generators possuem variáveis que determinam a maneira como os números aleatórios são criados. São eles:

- Tipo de generator: define o algoritmo de geração de números aleatórios. Atualmente há diversos tipos de generators - XORWOW, Philox, MRG32k3a e MTPG32 para números pseudo-aleatórios e SOBOL e SCRAMBLED-SOBOL para números quasi-aleatórios (figura 10).

- Semente, ou seed: inteiro de 64 bits que inicializa o estado inicial de um generator. A mesma semente produz sempre a mesma sequência de números aleatórios.

- Deslocamento, ou offset: define quantas posições serão deslocadas na sequência de números aleatórios. Isso permite que múltiplas execuções do mesmo programa continuem gerando resultados a partir da mesma sequência sem repetições.

- Ordem, ou order: define a maneira como os resultados vão ser alocados na memória global da GPU.

A diferença básica entre a implementação na CPU e na GPU é que, para o primeiro caso é utilizado um generator único, enquanto no segundo cada thread precisa do seu próprio generator. Todos os generators são capazes de criar sequências de números aleatórios respeitando distribuições uniformes, normais ou log-normais. Neste trabalho, utilizamos o generator XORWOW para a geração de sequências de números aleatórios distribuidos normalmente, com média 0 e desvio padrão 1. Este generator possibilita a criação de uma sequência de números aleatórios com um período maior que $2^{190}$.

\subsection{Setup}

Para esta implementação, foi utilizada a placa gráfica Nvidia GeForce GTX 750 Ti (figura 11) e a versão 8.0 do CUDA, baixada gratuitamente pelo site do fabricante. Além disso, 
Figura 10 - Comparação de desempenho entre os generatos cuRAND em número de geração de amostras por segundo

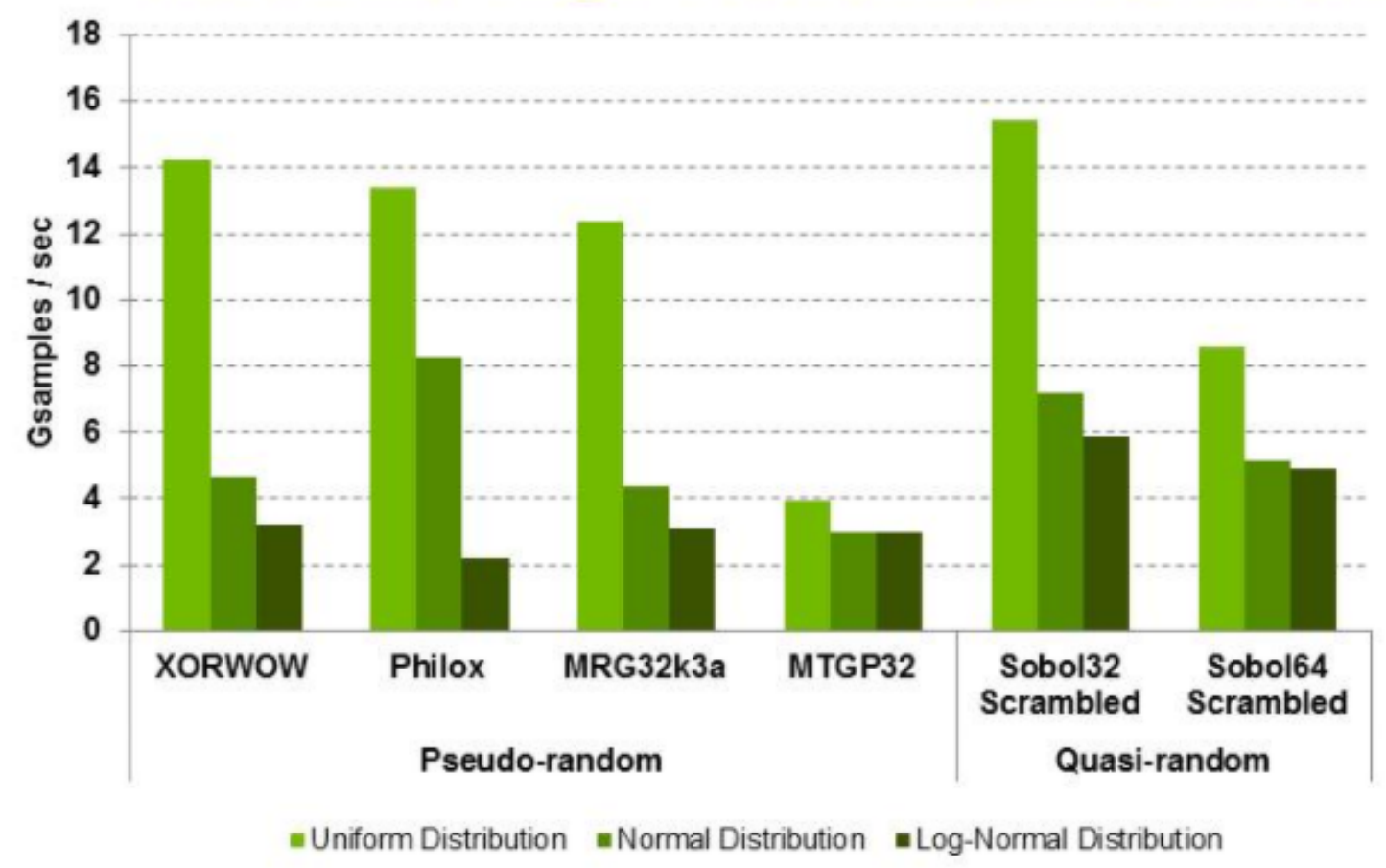

Fonte: (NVIDIA, 2016)

as especificações do dispositivo e da versão do CUDA podem ser checadas através da ferramenta deviceQuery, presente no toolkit CUDA (figura 12).

\subsection{Algoritmo}

O algoritmo executado na GPU está dividido em 2 etapas principais: geração dos números aleatórios e construção dos random walks para o cálculo do preço da opção. Esta divisão foi feita para fins de desempenho, conforme citado em seção anterior (cuRAND). Precisamos definir qual o número $N$ de threads utilizadas em cada etapa para minimizar o tempo de execução. 3 variáveis precisam ser levadas em conta:

- A - número de ativos envolvidos no cesto

- $P$ - número de passos de cada simulação

- $S$ - número de simulações na execução do programa

Para a otimização do setup e criação dos números aleatórios, precisamos minimizar o número de chamadas aos geradores e maximizar o bloco de números aleatórios gerados em 
Figura 11 - Especificações Nvidia GeForce GTX 750 Ti - Fabricante

GeForce GTX 750 Ti

\begin{tabular}{|c|c|}
\hline \multicolumn{2}{|c|}{ Especificações do mecanismo da GPU } \\
\hline Núcleos CUDA & 640 \\
\hline Base Clock & $1020 \mathrm{MHz}$ \\
\hline Boost Clock & $1085 \mathrm{MHz}$ \\
\hline \multicolumn{2}{|l|}{ Especificações de memória } \\
\hline Velocidade da memória ( Gbps) & 5,4 \\
\hline Quantidade de memória & $2048 \mathrm{MB}$ \\
\hline Interface de memória & 128-bit GDDR5 \\
\hline Largura de banda máxima & $86,4 \mathrm{~GB} / \mathrm{sec}$ \\
\hline \multicolumn{2}{|l|}{ Recursos da placa } \\
\hline FXAA and TXAA & $\checkmark$ \\
\hline 3D Vision & $\checkmark$ \\
\hline PhysX & $\checkmark$ \\
\hline Ambiente de programação & CUDA \\
\hline DirectX & 11.2 \\
\hline OpenGL & 4.4 \\
\hline Tipo de barramento & $\mathrm{PCl}-\mathrm{E} 3.0$ \\
\hline 3D Gaming & $\checkmark$ \\
\hline Blu Ray 3D & $\checkmark$ \\
\hline
\end{tabular}

\begin{tabular}{|c|c|}
\hline \multicolumn{2}{|l|}{ Especificações } \\
\hline Resolução Digital Máxima & $4096 \times 2160$ \\
\hline Resolução VGA máxima & $2048 \times 1536$ \\
\hline Conexão de mídia & $\begin{array}{l}\text { - Mini HDMI } \\
\text { - Dual Link DVI-D } \\
\text { - Dual Link DVI-I }\end{array}$ \\
\hline Vários monitores & $\checkmark$ \\
\hline $\mathrm{HDCP}$ & $\checkmark$ \\
\hline HDMI & $\checkmark$ \\
\hline Áudio HDMI & Internal \\
\hline \multicolumn{2}{|l|}{ Dimensões } \\
\hline Altura & $11.16 \mathrm{~cm} / 4.376$ Inches \\
\hline Comprimento & $14,48 \mathrm{~cm} / 5.7$ Inches \\
\hline Largura & Dual Slot \\
\hline \multicolumn{2}{|l|}{ Energia e temperatura } \\
\hline Temperatura máxima & $95 \mathrm{C}$ \\
\hline Consumo de energia & $60 \mathrm{~W}$ \\
\hline Requisito mínimo de alimentação & $300 \mathrm{~W}$ \\
\hline
\end{tabular}

Fonte: (NVIDIA, 2017)

cada chamada. Utilizamos então $N=A \times P \times S$, ou seja, uma thread para cada número aleatório gerado, conforme demonstrado na figura 13. Estes números são armazenados na memória global da GPU para depois serem acessadas pelas threads responsáveis pela construção dos random walks.

Para a simulação dos caminhos dos ativos, temos algumas restrições: não podemos paralelizar a geração dos passos de cada random walk pois cada passo depende do passo anterior para ser computado. Também não podemos paralelizar a contrução da trajetória de cada ativo dentro de uma mesma simulação pois, como os ativos estão correlacionados, a cada passo precisamos aplicar o efeito desta correlação aos random walks gerados. Para efeito de simplificação, neste algoritmo consideramos a matriz de correlações constante entre os ativos. Este fato isolado nos permitiria aumentar o nível de paralelização do código, mas tal efeito seria completamente artificial; logo, optamos por não utilizar deste artifício. 
Figura 12 - Especificações Nvidia GeForce GTX 750 Ti - deviceQuery

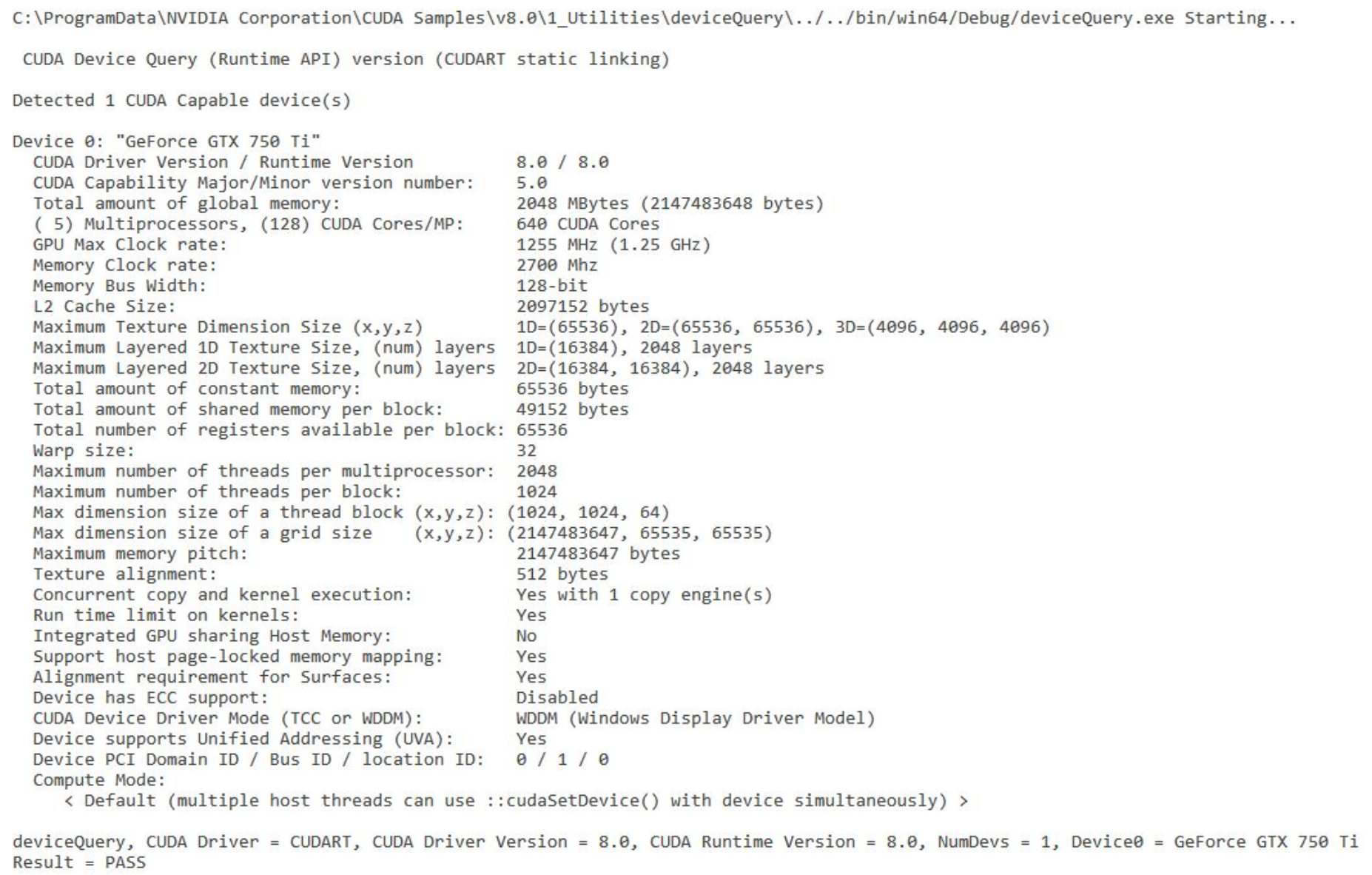

Fonte: produzido pelo autor.

Desta forma, conseguimos paralelizar nesta etapa apenas a execução de cada simulação, ou seja, $N=S$. A cada passo $p$ são calculadas as $p$-ésimas posições no random walk de cada ativo. As figuras 14, 15 e 16 ilustram de maneira simplificada a execução desta etapa do algoritmo.

Após a construção dos caminhos aleatórios, calculamos o preço de cada opção worst-of utilizando os preços finais dos ativos em cada uma das simulações. Os preços das worst-of's são então trazidos a valor presente e o preço final da opção é dado pela média destes, conforme discutido no capítulo Simulação. Desta forma, podemos enumerar o algoritmo através das seguintes etapas:

1. Cálculo das dimensões do grid a partir do número de ativos, número de simulações e número de passos em cada simulação;

2. Geração das variáveis aleatórias em paralelo (GPU); 
Figura 13 - Paralelização para a geração dos números aleatórios na GPU.

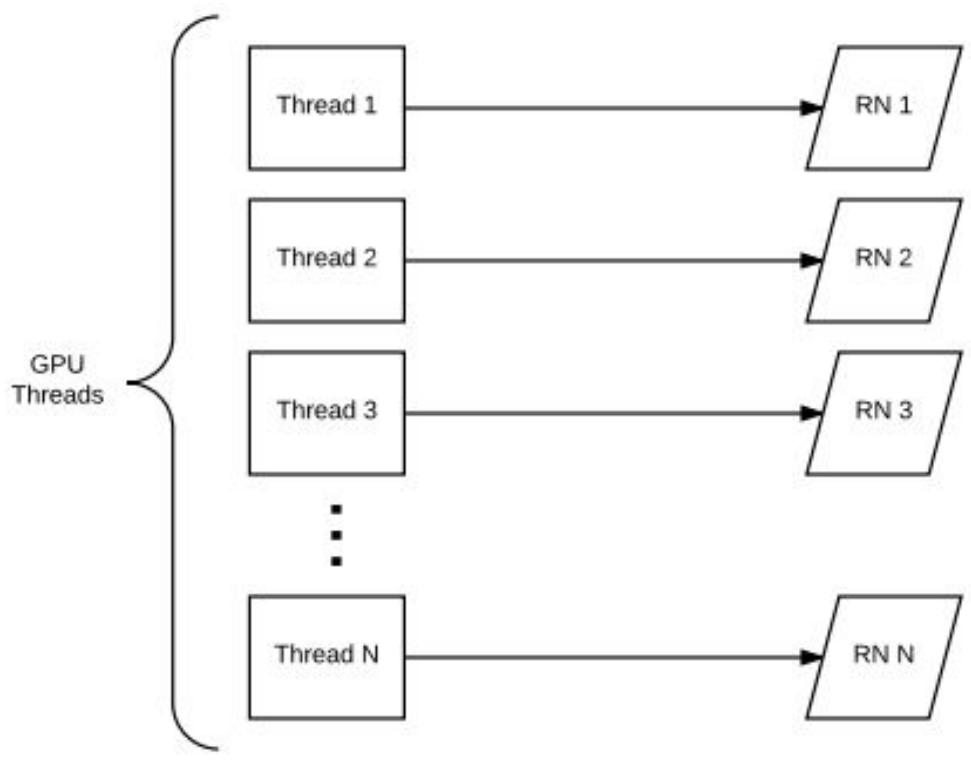

Fonte: produzido pelo autor.

Figura 14 - Paralelização para a construção dos random walks correlacionados na GPU - passo 1.

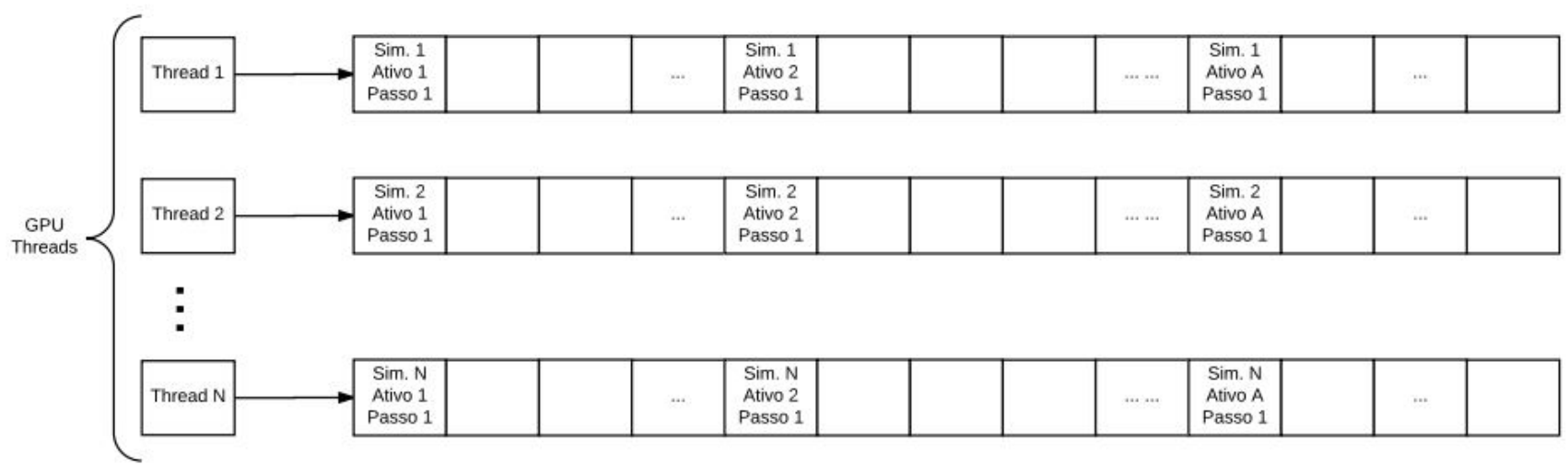

Fonte: produzido pelo autor.

3. Criação dos random walks e cálculo do payoff da opção no final de cada um deles, em paralelo (GPU);

- Utilização da Decomposição de Cholesky para correlacionar os random walks (apêndice $\mathrm{A})$.

4. Cálculo do valor final da opção. 
Figura 15 - Paralelização para a construção dos random walks correlacionados na GPU - passo 2.

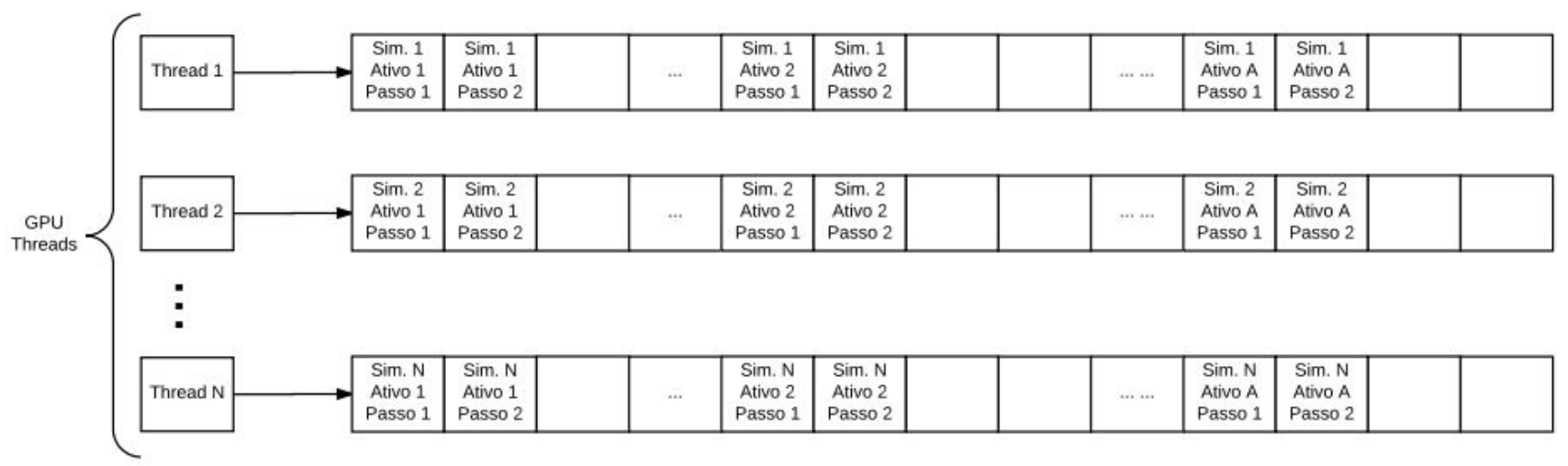

Fonte: produzido pelo autor.

Figura 16 - Paralelização para a construção dos random walks correlacionados na GPU - passo P.

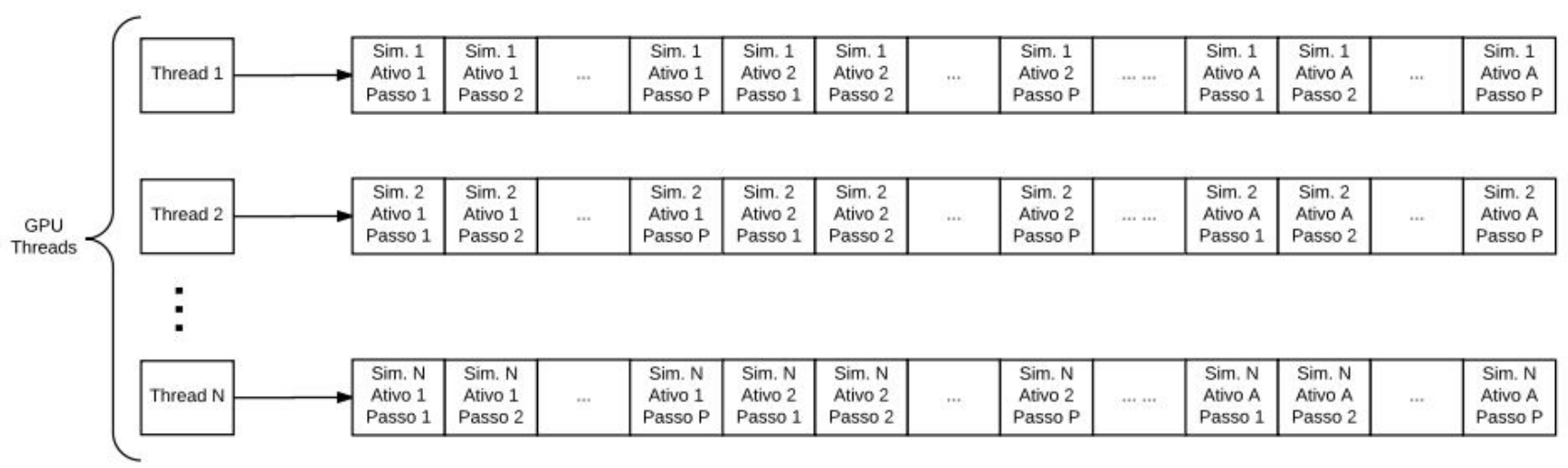

Fonte: produzido pelo autor.

O código-fonte completo utilizando CUDA pode ser consultado no apêndice B.

\subsection{Considerações Finais}

Neste capítulo foram discutidos os principais elementos da plataforma CUDA de computação paralela: histórico, arquitetura e desempenho. Também foram abordados a biblioteca cuRAND para a geração dos números aleatórios, bem como as configurações utilizada pelo autor e a lógica presente no algoritmo implementado neste trabalho. No capítulo seguinte serão apresentados os resultados de desempenho e preço em diversos cenários. 
Neste capítulo serão apresentados os resultados obtidos no projeto de maneira objetiva. Para todas as simulações, consideraremos sempre opções do tipo call com 90 dias de prazo (ou seja, cada simulação tem 90 passos), 2 ativos no cesto e o preço inicial de todos os ativos como 100 , bem como o strike da opção $\left(S_{0}=K=100\right)$. Será desconsiderada a taxa de juros $(r=0)$.

\subsection{Threads por bloco}

Uma das variáveis que pode influenciar no desempenho do algoritmo é o número de threads por bloco utilizado na execução de cada kernel. Na tabela 1 é possível observar o tempo de exeução do algoritmo para diferentes cenários de número de threads. O número de simulações utilizado em todos os cenários foi de $10^{5}$.

Tabela 1 - Tempo de execução dos algoritmos (segundos) para diferentes cenários de número de threads

\begin{tabular}{cc}
\hline Threads por bloco & Tempo de execução \\
\hline \hline 300 & 0,2506 \\
\hline 400 & 0,2463 \\
\hline 500 & 0,2513 \\
\hline 600 & 0,2511 \\
\hline 700 & 0,2378 \\
\hline 800 & 0,2423 \\
\hline 900 & 0,2406 \\
\hline 1024 & 0,2453 \\
\hline
\end{tabular}

Fonte - Produzido pelo autor.

Conforme observado, a variação no número de threads por bloco resultou apenas em 
variações marginais no tempo de execução do algoritmo. Em execuções diferentes com o mesmo número de threads, as mesmas variações podem ser observadas. Em outras palavras, não foi possível tirar nenhuma conclusão empírica sobre o número de threads por bloco.

\subsection{Desempenho do Algoritmo}

Esta é a principal seção de resultados neste trabalho. Para aferir estes resultados, foi produzido um código similar, porém executado de maneira sequencial. O código-fonte completo do algoritmo sequencial pode ser consultado no apêncide $C$.

Para a geração dos números aleatórios utilizamos o método Polar, descrito em (MARSAGLIA; BRAY, 1964). Escolhemos este método devido à sua boa performance computacional. Desta forma, executamos cada um dos algoritmos aumentando gradualmente o número de simulações, considerando sempre 2 ativos no cesto, com 0,5 de correlação e $20 \%$ de volatilidade.

Os tempos de execução são exibidos a seguir na tabela 2 e na figura 10.

Tabela 2 - Tempo de execução dos algoritmos (segundos) para diferentes cenários de número de simulações

\begin{tabular}{ccc}
\hline Simulações & Sequencial & CUDA \\
\hline \hline $10^{2}$ & 0,043 & 0,1074 \\
\hline $10^{3}$ & 0,044 & 0,1095 \\
\hline $10^{4}$ & 0,331 & 0,1198 \\
\hline $10^{5}$ & 2,967 & 0,2584 \\
\hline $10^{6}$ & 28,661 & 0,5516 \\
\hline \multicolumn{3}{c}{ Fonte - Produzido pelo autor. }
\end{tabular}

Pode-se facilmente observar que o algoritmo executado na GPU teve um desempenho muito superior ao algoritmo sequencial. Todas as execuções mantiveram-se abaixo de um segundo, que representa um tempo de resposta adequado para a solução proposta. Nos cenários com número de simulações baixo o algoritmo sequencial teve um desempenho superior, e isso pode ser explicado devido ao fato do algorito tradicional não utilizar a biblioteca $c u R A N D$ para a geração dos numeros aleatórios em paralelo. A alocação de memória para as variáveis aleatórias é a operação mais custosa do algoritmo em termos de tempo de processamento. 
Figura 17 - Tempo de execução das simulações executadas.

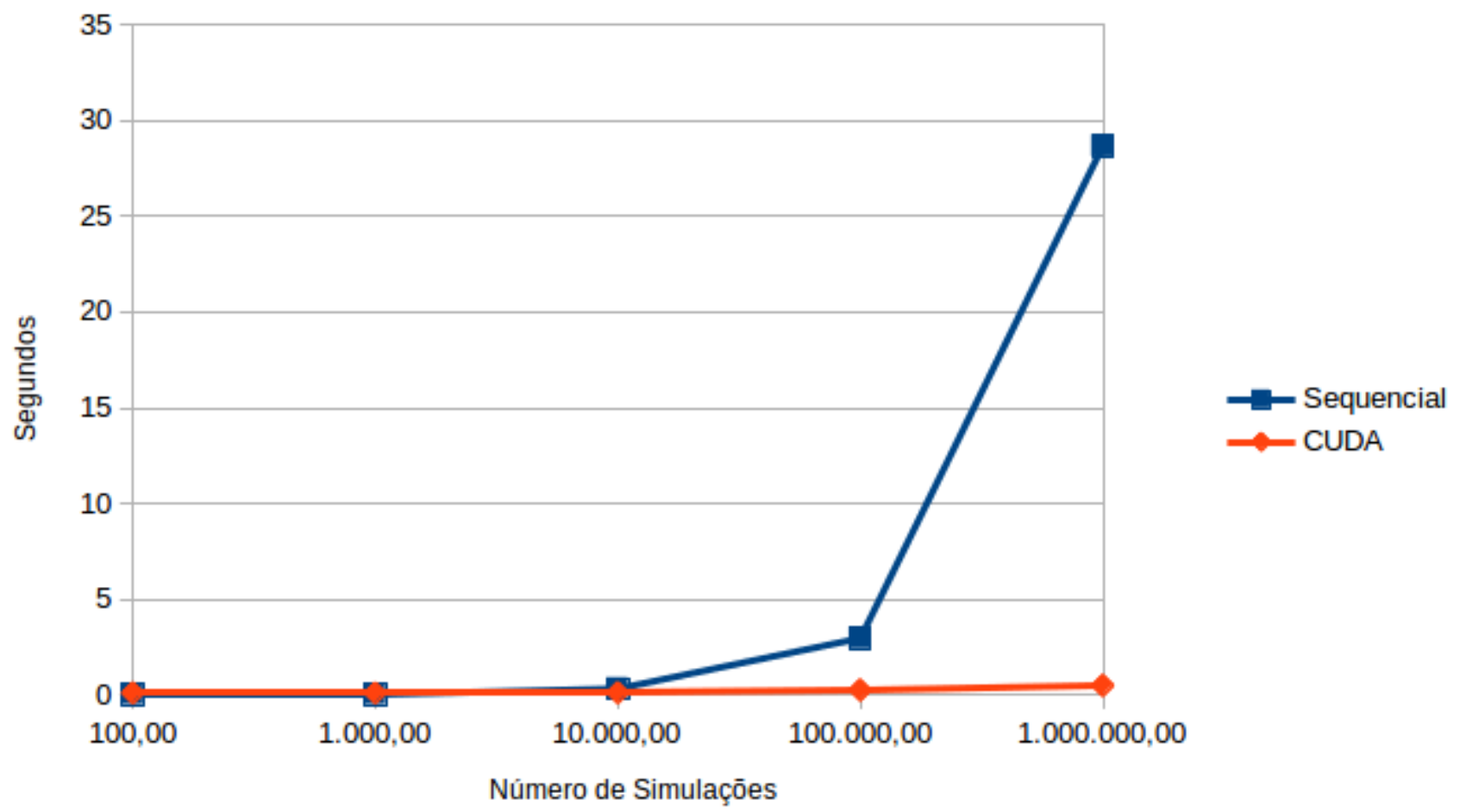

Fonte: produzido pelo autor. 



\section{CONCLUSÃO}

A proposta inicial deste trabalho foi a construção de uma ferramenta eficiente para a precificação em tempo real de ativos utilizando métodos de simulação numérica. O objetivo final consistia em obter resultados instantâneos para a precificação de contratos complexos no mercado de derivativos, cujo maior gargalo é o tempo de processamento necessário durante as simulações de preços.

A solução adotada foi uma implementação paralela da simulação Monte Carlo. Devido a fatores como custo e esforço de implementação, foi escolhida a plataforma CUDA de computação paralela para a precificação de opções multivariadas do tipo worst-of. Foram discutidos os conceitos básicos da precificação analítica de opções e do comportamento das variáveis aleatórias ao longo do tempo. Foram demonstradas a existência de medidas equivalentes de probabilidade e a não-existência de arbitragem para garantir uma precificação consistente. Além disso, foram apresentados os conceitos centrais da simulação Monte Carlo, bem como os principais elementos e as características mais relevantes da arquitetura CUDA.

Através da análise dos resultados, podemos concluir que a implementação da calculadora utilizando a paralelização via GPU mostrou-se uma ótima escolha. $\mathrm{O}$ código executado na GPU apresentou um desempenho superior ao código convencional, conforme esperado. Para os casos com poucas simulações, o algoritmo sequencial teve desempenho superior, e isso pode ser explicado devido ao custo de tempo para alocação de memória para a criação das variáveis aleatórias quando utilizamos a biblioteca $c u R A N D$.

É importante mencionar que a implementação das opções worst-of representa apenas um dentre os diversos exemplos de produtos possíveis. A solução é escalável e facilmente adaptável: condicionado à adição das funções específicas de payoff de cada produto, é possível garantir preço praticamente instantâneo para qualquer estrutura negociada. Ou seja, com um pequeno nível de esforço, a solução é facilmente expansível.

As principais limitações deste trabalho encontram-se na modelagem dos demais elemen- 
tos presentes na precificação, como a volatilidade e as taxas de juros. Estes elementos foram considerados constantes neste estudo e seus respectivos modelos serão abordados em trabalhos futuros, onde será possível refinar a simulação numérica. 


\section{REFERÊNCIAS}

ANDERSEN, L.; PITERBARG, V. Interest rate modeling-volume iii: Products and risk management. Atlantic Financial Press, v. 1, p. 2, 2010. Citado na página 41.

BJÖRK, T. Arbitrage theory in continuous time. [S.1.]: Oxford university press, 2009. Citado na página 40.

BLACK, F.; SCHOLES, M. The pricing of options and corporate liabilities. Journal of political economy, The University of Chicago Press, v. 81, n. 3, p. 637-654, 1973. Citado na página 36.

BROWN, R. A Brief Account of Microscopical Observations Made... on the Particles Contained in the Pollen of Plants, and on the General Existence of Active Molecules in Organic and Inorganic Bodies. [S.1.: s.n.], 1828. Citado na página 29.

CARREIRA, M. C.; JR, R. J. B. Brazilian Derivatives and Securities: Pricing and Risk Management of FX and Interest-Rate Portfolios for Local and Global Markets. [S.1.]: Springer, 2016. Nenhuma citação no texto.

CLARK, I. J. Foreign exchange option pricing: a practitioner's guide. [S.1.]: John Wiley \& Sons, 2011. Citado na página 45.

CLUA, E. W. G.; ZAMITH, M. P. Programming in cuda for kepler and maxwell architecture. Revista de Informática Teórica e Aplicada, v. 22, n. 2, p. 233-257, 2015. Citado na página 54.

CONTINUUM. Monte Carlo Asset Paths. 2017. Disponível em: <https://www.continuum.io/ sites/default/files/montecarloplot.png>. Citado na página 48.

COX, J. C.; JR, J. E. I.; ROSS, S. A. An intertemporal general equilibrium model of asset prices. Econometrica: Journal of the Econometric Society, JSTOR, p. 363-384, 1985. Citado na página 37.

DELTAQUANTS. Risk Analysis of Worst-of and Best-of Options. 2016. Disponível em: $<$ http://www.deltaquants.com/risk-analysis-of-worst-of\%20and-best-of-options $>$. Citado na página 46.

ESPOSITO, F. P. Multidimensional black-scholes options. 2010. Citado na página 38.

FRANKLIN, B. Advice to a young tradesman (1748). George Fisher: The American Instructor: or Young Man's Best Companion.... The Ninth Edition Revised and Corrected. Philadelphia: Printed by B. Franklin and D. Hall, at the New-Printing-Office, in MarketStreet, p. 375-7, 1748. Citado na página 25.

GALlOY, M. CPU vs GPU Performance. 2013. Disponível em: < http://michaelgalloy.com/ 2013/06/11/cpu-vs-gpu-performance.html>. Citado na página 53.

HANZON, B.; SPIEKSMA, F. Pricing derivatives on multiple assets. 2013. Citado na página 45. 
HULL, J. C. Options, futures, and other derivatives. [S.1.]: Pearson Education India, 2006. Nenhuma citação no texto.

IANCU, A. K. Numerical methods for pricing basket options. Tese (Doutorado) - The Ohio State University, 2004. Citado na página 45.

JACOD, J.; PROTTER, P. Probability essentials. [S.1.]: Springer Science \& Business Media, 2004. Citado na página 30.

KIRK, D. B.; WEN-MEI, W. H. Programming massively parallel processors: a hands-on approach. [S.1.]: Morgan Kaufmann, 2016. Citado na página 52.

MARSAGLIA, G.; BRAY, T. A. A convenient method for generating normal variables. Siam Review, SIAM, v. 6, n. 3, p. 260-264, 1964. Citado na página 64.

MÖRTERS, P.; PERES, Y. Brownian motion. [S.1.]: Cambridge University Press, 2010. v. 30. Citado na página 32.

MUIRHEAD, S.; BOROVKOV, K. Financial Geometry: Pricing multi-asset barrier options using the generalised reflexion principle. Tese (Doutorado) - Ph. D. Thesis, 2011, University of Melbourne, 2011. Citado na página 45.

MUSIELA, M.; RUTKOWSKI, M. Martingale methods in financial modelling. [S.1.]: Springer Science \& Business Media, 2006. v. 36. Citado na página 40.

NICKOLLS, J.; DALLY, W. J. The gpu computing era. IEEE micro, IEEE, v. 30, n. 2, 2010. Citado na página 51.

NVIDIA. cuRAND guide. 2016. Disponível em: <https://developer.nvidia.com/curand>. Citado na página 58 .

CUDA Parallel Computing Platform. 2017. Disponível em: <http://www.nvidia.com>. Citado nas páginas 52, 55, 56 e 59 .

ØKSENDAL, B. Stochastic differential equations. In: Stochastic differential equations. [S.1.]: Springer, 2003. p. 65-84. Citado na página 32.

SENTHILNATH, J.; RAJ, A.; OMKAR, S.; MANI, V.; KUMAR, D. Quasi-based hierarchical clustering for land cover mapping using satellite images. SPRINGER-VERLAG BERLIN, 2013. Citado na página 50.

SHREVE, S.; KARATZAS, I. Brownian motion and stochastic calculus. Newyork Berlin. Heidelberg. London Paris Tokyo, 1991. Citado nas páginas 32 e 35.

WORST-OF Option. Disponível em: <http://www.investment-and-finance.net/derivatives/w/ worst-of-option.html>. Citado na página 46. 
APÊNDICE

\section{A}

\section{DECOMPOSIÇÃO DE CHOLESKY}

No algoritmo, para aplicar o valor da correlação durante a construção dos random walks, foi utilizada a decomposição de Cholesky. Este método, da álgebra linear, decompõe uma matriz hermitiana positiva-definida $A$ no produto de uma matriz triangular inferior $L$ e sua conjugada transposta $L^{*}$.

$$
A=L L^{*}
$$

O termo hermitiana, de maneira resumida, representa uma extensão do termo simétrica para o contexto dos números complexos (não utilizados neste trabalho). Uma matriz hermitiana positiva-definida $M$, além de simétrica, apresenta o escalar $z^{T} M z$ positivo para qualquer coluna não-nula $z$ de $n$ números reais.

A cada iteração de cada simulação, primeiramente calculamos o vetor $W_{n}$ de números aleatórios independentes, sendo $n$ o número de ativos que compõe o cesto. Após isso, calcula-se o vetor $Z_{n}$ através da multiplicação do vetor $W_{n}$ pela matriz $L$, que representa a matriz triangular inferior obtida através da decomposição de Cholesky da matriz de correlação $n \times n$ dos ativos.

Nesta implementação, assumimos constantes as correlações entre os ativos, logo a decomposição de Cholesky é realizada uma única vez, no início do algoritmo. No caso de correlações variáveis, a matriz precisa ser atualizada e decomposta novamente a cada iteração do algoritmo. 

APÊNDICE

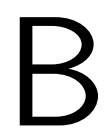

CÓDIGO-FONTE CUDA

Código-fonte 1 - Código fonte CUDA

1: \#include <stdio.h>

2: \#include <stdlib.h>

3: \#include <math.h>

4: \#include <omp.h>

5: \#include <curand_kernel.h>

6: \#include <curand.h>

7: \#include "cuda_runtime.h"

8: \#include "device_launch_parameters.h"

9: \#include <time.h>

10 :

11: \#define N_ATV 2

12: \#define $\mathrm{N}_{-}$STEPS 90

13: \#define $\mathrm{N}_{-}$SIM 1000

14: \#define BLOCKSIZE 1024

15:

16: // Payoff() > retorna o payoff opcao

17: __device_ float Payoff

18: char tipo, $/ / c "-c a l l o u " p "-p u t$

19: float S, // ativo

20: float K) $\{$ // strike

21:

22: int phi $=1$;

23: if (tipo $==$ ' $p$ ') \{

24: $\quad$ phi $=-1$;

25: \}

26: 
27: if $((S-K)>0)\{$

28: return phi $*(\mathrm{~S}-\mathrm{K})$;

29: \}else

30: return 0.0 ;

31: \}

32: $\}$

33:

34:

35: // Worstof() > retorna o payoff da opcao "worst of"

36: __device__ float Worstof (

37: int atv,

38: float $* \mathrm{~S}$,

39: float $\mathrm{K}$,

40: $\operatorname{char} *$ tipo) \{

41:

42: int $i$;

43: float preco $=0$;

44: float atual $=0$;

45:

46: for $(i=0 ; i<\operatorname{atv} ; i++)\{$

47: atual $=$ Payoff(tipo[i], $S[i], K)$;

48: if $(i==0)\{$

49:

50: $\quad$ preco $=$ atual

51:

52: \}

53:

54: else if (atual < preco) \{

55: $\quad$ preco = atual;

56: \}

57: $\}$

58:

59: if $($ preco<0 \{

60: $\quad$ preco $=0$;

61: \}

62:

63: return preco;

64:

65: $\}$

66:

67: 
68: // cholesky() > retorna a matriz triangular inferior resultante da decomposicao de Cholesky

69: float *cholesky(float *A, int $\mathrm{n}$ ) \{

70:

71: int $i, j, k$;

72: float $* \mathrm{~L}=($ float $*)$ calloc $(\mathrm{n} * \mathrm{n}$, sizeof (float));

73: if ( $\mathrm{L}==\mathrm{NULL})$

74: $\quad$ return 0 ;

75:

76: for $(i=0 ; i<n ; i++)$

77: $\quad$ for $(j=0 ; j<(i+1) ; j++)\{$

78: $\quad$ float $s=0$;

79: $\quad$ for $(k=0 ; k<j ; k++)$

80: $\quad \mathrm{s}+=\mathrm{L}[\mathrm{i} * \mathrm{n}+\mathrm{k}] * \mathrm{~L}[\mathrm{j} * \mathrm{n}+\mathrm{k}]$;

81:L L $[i * n+j]=(i==j) ? \operatorname{sqrtf}(A[i * n+i]-s):(1.0 /$ $\mathrm{L}[j * \mathrm{n}+\mathrm{j}] *(\mathrm{~A}[\mathrm{i} * \mathrm{n}+\mathrm{j}]-\mathrm{s}))$;

82: $\quad\}$

83: $\quad$ return L;

84:

85: $\}$

86:

87:

88: // multiplicacao de matrizes

89: __device__ void matrix_mult (float $* A$, float $* B$, int $n$, float $* C$ ) \{

90:

91: int i, $j$;

92: float sum;

93:

94: for $(i=0 ; i<n ; i++)\{$

95: $\quad$ sum $=0.0$;

96: $\quad$ for $(j=0 ; j<n ; j++)\{$

97: $\quad$ sum $=\operatorname{sum}+(A[i *(n-1)+j] * B[j])$;

98: \}

99: $\quad C[i]=$ sum;

100: \}

101:

102: $\}$

103:

104:

105: // setup para a geracao dos numeros aleatorios 
106: __global__ void setupRN_kernel (curandState *state, const unsigned long long seed, unsigned long long $n$ ) \{

107:

108: unsigned long long id = threadIdx. $x+$ blockIdx.x*blockDim.x;

109:

110: if $(i d<n)\{$

111: curand_init $($ seed $<20)+i d, 0$, 0 , \&state[id]);

112:

113: \}

114:

115: \}

116:

117:

118: // kernel para geracao dos random paths

119: __global__ void generateRandomPath (float *paths, float *finals, float *worst, char *tipo, float *SO, float K, float *vol, float H, float *correlDec, curandState *rng \{

120:

121: int id $=$ threadIdx.x + blockIdx.x*blockDim. $x$;

122: curandstate localstate;

123:

124: if $\left(\right.$ id $<N_{-}$SIM) \{

125: int $i, j=0$;

126: float $W\left[N_{-}\right.$ATV ] ;

127: float Z[N_ATV];

128: float $S_{-} T\left[N_{-} A T V\right]$;

129:

130: for $\left(i=0 ; i<N_{-}\right.$STEPS; $\left.i++\right)\{$

131: for $\left(j=0 ; j<N_{-}\right.$ATV $\left.; j++\right)\{$

132: $\quad$ localstate $=\operatorname{rng}\left[i+j * N_{-}\right.$STEPS $+i d * N_{-}$STEPS $* N_{-}$ATV $]$;

133: $\quad W[j]=$ curand_normal (\&localstate);

134: $\quad\}$

135:

136: $\quad$ matrix_mult (W, correlDec, N_ATV, Z);

137:

138: $\quad$ if $(i==0)\{$

139: $\quad$ for $\left(j=0 ; j<N_{-}\right.$ATV $\left.; j++\right)\{$

140: $\quad$ paths $\left[i+j * N_{-}\right.$STEPS $+i d * N_{-}$STEPS $* N_{-}$ATV $]=S 0[j]$;

141: $\quad\}$

142: $\quad\}$

143: else\{

144: $\quad$ for $\left(j=0 ; j<N_{-}\right.$ATV $\left.; j++\right)\{$ 
145:

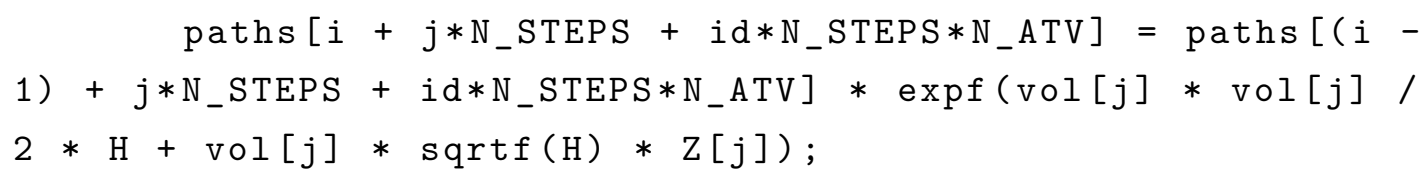

146:

147:

if $\left(i==\left(N_{-}\right.\right.$STEPS -1$\left.)\right)\{$

148:

$S_{-} T[j]=$ paths $\left[i+j * N_{-}\right.$STEPS $+i d * N_{-}$STEPS $* N_{-}$ATV $]$;

149: finals $\left[\left(N_{-}\right.\right.$ATV *id $\left.)+j\right]=S_{-} T[j]$;

150:

151:

if $\left(j==\left(N_{-}\right.\right.$ATV -1$\left.)\right)\{$

152: worst $[i d]=$ Worstof $\left(N_{-} A T V, S_{-} T, K\right.$, tipo);

153: \}

154:

155: $\quad\}$

156:

157: $\quad\}$

158:

159: $\quad\}$

160:

161: \}

162:

163: \}

164:

165: \}

166:

167:

168: \#define gpuErrchk(ans) \{ gpuAssert((ans), _FILE__, __LINE__ ); \}

169: inline void gpuAssert(cudaError_t code, const char *file, int line, bool abort = true)

170: \{

171: if (code $!=$ cudaSuccess) \{

172: $\quad$ printf ("GPUassert: $\% \mathrm{~s} \% \mathrm{~s} \% \mathrm{~d} \backslash \mathrm{n}$ ", cudaGetErrorString(code), file, line);

173: if (abort) \{

174: $\quad$ cudaDeviceReset () ;

175: $\quad$ exit (code) ;

176: \}

177:

178: \}

179:

180: $\}$

181: 
182:

183: int main(int $\operatorname{argc}$, char *argv[])

184: \{

185:

186: // variaveis

187: clock_t begin = $\operatorname{clock}()$;

188: curanditate *d_States;

189: float *d_Paths, *h_Paths, *d_Finals, *h_Finals, *d_Worst, * h_Worst;

190: float $* d_{-}$SO, *d_vol, *d_L ;

191: char *d_tp;

192:

193: float $T=1.0$;

194: float $r=0.0$;

195: float $K=100.0$;

196: float $\mathrm{h}_{-} \mathrm{SO}[]=\{100.0,100.0\}$;

197: $\quad$ char $* h_{-} t p=" c c "$;

198: float $h_{-} \operatorname{vol}[]=\{0.2,0.3\}$;

199: float $h_{-} \operatorname{corr}[]=\{1,0.5$,

200: $\quad 0.5,1\}$;

201:

202: // escolhe uma semente aleatoria

203: $\quad$ srand (time (NULL));

204: unsigned int seed = rand ();

205:

206: // define o tamanho do grid

207: unsigned long $\mathrm{n}=\mathrm{N}_{-}$ATV $* \mathrm{~N}_{-}$STEPS $* \mathrm{~N}_{-}$SIM ;

208: int nBlocks $=\mathrm{n} /$ BLOCKSIZE $+(\mathrm{n} \% \mathrm{BLOCKSIZE}=0$ ? $0: 1)$;

209: printf("\npt1 - n: \%d, blockSize: \%d, nBlocks: \%d, seed: \%d $\backslash n$ ", n, BLOCKSIZE, nBlocks, seed);

210:

211: // kernel para setup das variaveis aleatorias

212: gpuErrchk (cudaMalloc((void **) \&d_States, n*sizeof (curandState )));

213: setupRN_kernel $<<$ nBlocks, BLOCKSIZE $\gg>\left(d_{-}\right.$States, seed, $\mathrm{n}$ );

214: gpuErrchk (cudaPeekAtLastError ()) ;

215: gpuErrchk (cudaDeviceSynchronize ()) ;

216:

217: // define o numero de blocos

218: $\quad$ int $\mathrm{n} 2=\mathrm{N}_{-} \mathrm{SIM}$;

219: nBlocks $=\mathrm{n} 2 /$ BLOCKSIZE $+(\mathrm{n} 2 \%$ BLOCKSIZE $=0 ? 0: 1)$; 
220: printf("\npt2 - n: \%d, blockSize: \%d, nBlocks: \%d \n", n2, BLOCKSIZE, nBlocks);

221:

222: // decomposicao de cholesky na matriz de correlacoes

223: float $* h_{-} L=\operatorname{cholesky}\left(h_{-} \operatorname{corr}, N_{-}\right.$ATV);

224:

225: // tamanho do "passo"

226: float $\mathrm{H}=\mathrm{T} / \mathrm{N}_{-}$STEPS ;

227:

228: I/ aloca as variaveis

229: gpuErrchk (cudaMalloc ( (void **) \&d_tp, N_ATV*sizeof (char))) ;

230: $\quad$ gpuErrchk (cudaMalloc ( (void **) \&d_SO, N_ATV*sizeof (float)));

231: gpuerrchk (cudaMalloc ( void **) \&d_vol, N_ATV*sizeof(float))) ;

232: gpuErrchk (cudaMalloc ( (void **) \&d_L, N_ATV*N_ATV*sizeof (float) ));

233:

234: // copia para device

235: gpuErrchk (cudaMemcpy (d_tp, h_tp, N_ATV*sizeof (char), cudaMemcpyHostToDevice)) ;

236: gpuErrchk (cudaMemcpy (d_SO, h_SO, N_ATV*sizeof (float), cudaMemcpyHostToDevice)) ;

237: gpuErrchk(cudaMemcpy (d_vol, h_vol, N_ATV*sizeof(float), cudaMemcpyHostToDevice)) ;

238: gpuErrchk (cudaMemcpy (d_L, h_L, N_ATV*N_ATV*sizeof (float), cudaMemcpyHostToDevice)) ;

239:

240: // aloca variaveis

241: gpuErrchk(cudaMalloc((void **) \&d_Paths, n*sizeof(float))) ;

242: gpuErrchk (cudaMalloc ( (void **) \&d_Finals, N_SIM*N_ATV*sizeof ( float)));

243: gpuErrchk (cudaMalloc ( (void **) \&d_Worst, N_SIM*sizeof(float)) ) ;

244:

245: // kernel para geracao dos caminhos aleatorios

246: generateRandomPath << <Blocks, BLOCKSIZE >> > d_Paths, d_Finals, d_Worst, d_tp, d_sO, K, d_vol, H, d_L, d_States);

247: gpuErrchk (cudaPeekAtLastError());

248: gpuErrchk (cudaDeviceSynchronize()) ;

249:

250: // copia as variaveis da GPU para a memoria do host

251: $\quad$ h_Paths $=($ float $*) \operatorname{malloc}(n * \operatorname{sizeof}($ float $))$;

252: $\quad h_{-}$Finals $=($float $*)$ malloc $\left(N_{-}\right.$SIM $* N_{-}$ATV $*$ sizeof (float) $)$; 
253: $\quad h_{-}$Worst $=($float $*$ ) malloc (N_SIM*sizeof (float) $)$;

254: gpuErrchk (cudaMemcpy (h_Paths, d_Paths, n*sizeof (float), cudaMemcpyDeviceToHost)) ;

255: gpuErrchk (cudaMemcpy (h_Finals, d_Finals, N_SIM*N_ATV*sizeof ( float), cudaMemcpyDeviceToHost));

256: gpuErrchk (cudaMemcpy (h_Worst, d_Worst, N_SIM*sizeof (float), cudaMemcpyDeviceToHost)) ;

257:

258: // Resultado final

259: float sum $=0$;

260: for (int $i=0 ; i<N_{-}$SIM; $i++$ ) \{

261: $\quad$ sum $+=h_{-}$Worst $[i]$;

262: \}

263:

264: // tempo de execucao

265: clock_t end $=\operatorname{clock}()$;

266: float time_elapsed $=$ (float) (end - begin) / CLOCKS_PER_SEC;

267:

268: // exibe output

269: printf("\nOption value: \%f $\backslash n$ ", ( $\left.\operatorname{sum} / \mathrm{N}_{-} \mathrm{SIM}\right) * \exp (-\mathrm{r} * \mathrm{~T})$ );

270: printf("Total time elapsed: $\%$ f $s \backslash n "$, time_elapsed);

271:

272: cudaDeviceReset ();

273: return 0 ;

274: $\}$ 
APÊNDICE

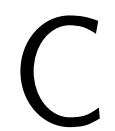

CÓDIGO-FONTE SEQUENCIAL

Código-fonte 2 - Código fonte Sequencial

1:

2: \#include <stdio.h>

3: \#include <stdlib.h>

4: \#include <math.h>

5: \#include <omp.h>

6: \#include "device_launch_parameters.h"

7: \#include <time.h>

8:

9: \#define N_ATV 2

10: \#define N_STEPS 90

11: \#define N_SIM 1000

12:

13: float Payoff (

14: char tipo, $\quad / / c "-c a l l o u$ "p" - put

15: float $S, \quad / /$ ativo

16: float K)\{ // strike

17:

18: int phi $=1$;

19: if (tipo $==$ ' $p$ ') \{

20: phi $=-1$;

21: $\quad\}$

22:

23: if $((S-K)>0)\{$

24: $\quad$ return phi $*(S-K)$;

25: $\quad\}$

26: else\{ 
27: $\quad$ return 0.0 ;

28: $\quad\}$

29: $\}$

30:

31: float Worstof (

32: int atv,

33: float $* \mathrm{~S}$,

34: float $K$,

35: char *tipo) \{

36:

37: int i;

38: float preco $=0$;

39: float atual $=0$;

40:

41: for $(i=0 ; i<\operatorname{atv;~} i++)\{$

42: atual = Payoff(tipo[i], $S[i], K)$;

43: if $(i==0)\{$

44: $\quad$ preco $=$ atual;

45: $\}$

46: else if (atual < preco) \{

47: $\quad$ preco = atual;

48: $\quad\}$

49: $\}$

50:

51: if $($ preco<0 \{

52: $\quad$ preco $=0$;

53: \}

54:

55: return preco;

56: $\}$

57:

58: void matrix_mult (float $* A$, float $* B$, int $n$, float $* C$ ) \{ 59:

60: int i, $j$;

61: float sum;

62:

63: for $(i=0 ; i<n ; i++)\{$

64: $\quad$ sum $=0.0$;

65: for $(j=0 ; j<n ; j++)\{$

66: $\quad \operatorname{sum}=\operatorname{sum}+(A[i *(n-1)+j] * B[j])$;

67: \}

68: $\quad C[i]=$ sum; 
69: $\quad\}$

70:

71: $\}$

72:

73:

74: float *cholesky(float *A, int $n)\{$

75:

76: int $i, j, k$;

77: float $* \mathrm{~L}=($ float $*) \operatorname{calloc}(\mathrm{n} * \mathrm{n}$, sizeof (float));

78: if ( $\mathrm{L}==\mathrm{NULL})$

79: $\quad$ return 0 ;

80:

81: for ( $i=0 ; i<n ; i++)$

82: $\quad$ for $(j=0 ; j<(i+1) ; j++)\{$

83: $\quad$ float $s=0$;

84: $\quad$ for $(k=0 ; k<j ; k++)$

$85:$

$$
\mathrm{s}+=\mathrm{L}[\mathrm{i} * \mathrm{n}+\mathrm{k}] * \mathrm{~L}[\mathrm{j} * \mathrm{n}+\mathrm{k}] \text {; }
$$

86:

87: $\mathrm{L}[i * \mathrm{n}+j]=(i=j) ? \operatorname{sgrtf}(\mathrm{A}[\mathrm{i} * \mathrm{n}+\mathrm{i}]-\mathrm{s}):(1.0$

$$
/ \mathrm{L}[j * \mathrm{n}+\mathrm{j}] *(\mathrm{~A}[\mathrm{i} * \mathrm{n}+\mathrm{j}]-\mathrm{s})) \text {; }
$$

88: $\quad\}$

89:

90: return L;

91: $\}$

92:

93: double randn(double mu, double sigma)

94: \{

95:

96: double U1, U2, W, mult;

97: $\quad$ static double X1, X2 ;

98: $\quad$ static int call $=0$;

99:

100: if $(\operatorname{call}==1)$

101: \{

102: $\quad$ call $=$ ! call;

103: $\quad$ return $(m u+\operatorname{sigma} *($ double $) \times 2)$;

104: \}

105:

106: do

107: \{

108: $\quad \mathrm{U} 1=-1+(($ double $)$ rand ()$/$ RAND_MAX $) * 2$;

109: $\quad \mathrm{U} 2=-1+(($ double $)$ rand ()$/$ RAND_MAX $) * 2$; 
110: $\quad W=\operatorname{pow}(U 1,2)+\operatorname{pow}(U 2,2)$;

111: \} while ( $>=1|| W==0)$;

112:

113: $\operatorname{mult}=\operatorname{sqrt}((-2 * \log (W)) / W)$;

114: $\mathrm{X} 1=\mathrm{U} 1 *$ mult;

115: $\mathrm{X} 2=\mathrm{U} 2 *$ mult;

116: $\operatorname{call}=$ ! call;

117:

118: $\operatorname{return}(\mathrm{mu}+\operatorname{sigma} *($ double $) \mathrm{X} 1)$;

119: \}

$120:$

121:

122: void generateRandomPath(float *paths, float *finals, float * worst, char *tipo, float *SO, float K, float *vol, float H, float *orrelDec) \{

123:

124: long id;

125: for (id $=0$; id $<N_{-}$SIM; $\left.i d++\right)\{$

126: int $i, j=0$;

127: float $W\left[N_{-}\right.$ATV $]$;

128: float Z[N_ATV];

129: float $S_{-}$T [N_ATV];

130:

131: for $\left(i=0 ; i<N_{-}\right.$STEPS; $\left.i++\right)\{$

132: $\quad$ for $\left(j=0 ; j<N_{-} A T V ; j++\right)\{$

133: $\quad W[j]=\operatorname{randn}(0,1)$;

134: \}

135:

136:

137:

138:

139:

140:

141:

142:

143:

144:

matrix_mult(W, correlDec, N_ATV, Z);

$145:$

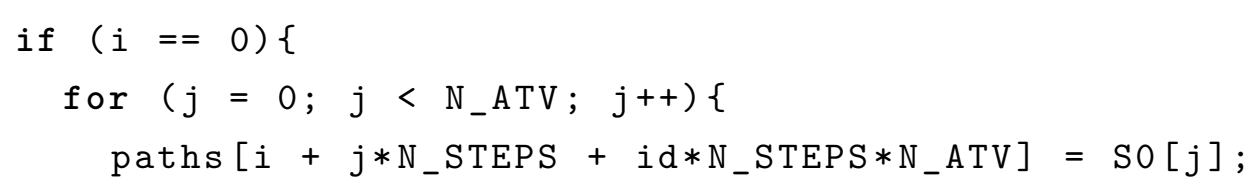

146:

147: 
148:

$S_{-} T[j]=$ paths $\left[i+j * N_{-}\right.$STEPS $+i d * N_{-}$STEPS $* N_{-}$ATV $]$;

149: finals $\left[\left(N_{-} A T V * i d\right)+j\right]=S_{-} T[j]$;

150: if $\left(j==\left(N_{-} A T V-1\right)\right)\{$

151: worst $[i d]=$ Worstof (N_ATV, S_T, K, tipo);

152: \}

153: $\quad\}$

154: $\quad\}$

155: $\quad\}$

156: \}

157: \}

158: \}

159:

160:

161: int main(int $\operatorname{argc,}$ char *argv[])

162: \{

163:

164: // variaveis

165: clock_t begin $=\operatorname{clock}()$;

166: float *h_Paths, *h_Finals, *h_Worst;

167: float $\mathrm{T}=1.0$;

168: float $r=0.0$;

169: float $K=100.0$;

170: float $h_{-} S O[]=\{100.0,100.0\}$;

171: $\quad$ char *h_tp $=$ "cc";

172: float $h_{-} \operatorname{vol}[]=\{0.2,0.2\}$;

173: float $h_{-} \operatorname{corr}[]=\{1,0.5$,

174: $\quad 0.5,1\}$;

175:

176: // parte 1 - setup e geracao de numeros aleatorios na GPU

177: long $\mathrm{n}=\mathrm{N}_{-}$ATV $* \mathrm{~N}_{-}$STEPS $* \mathrm{~N}_{-}$SIM

178: $\quad$ printf ("\npt1 - n: \%d \n", n);

179:

180: // decomposicao de cholesky na matriz de correlacoes

181: float $* h_{-} L=\operatorname{cholesky}\left(h_{-} \operatorname{corr}, N_{-}\right.$ATV $)$;

182:

183: // tamanho do "passo"

184: float $\mathrm{H}=\mathrm{T} / \mathrm{N}_{-} \mathrm{STEPS}$;

185:

186: // aloca variaveis para construcao dos caminhos aleatorios na memoria da GPU

187: $\quad$ h_Paths $=($ float $*) \operatorname{calloc}(n, \operatorname{sizeof}($ float $))$;

188: $\quad h_{-}$Finals $=$(float $*$ ) calloc $\left(N_{-}\right.$SIM $* N_{-} A T V$, sizeof (float)) ; 
189: $\quad h_{\text {_Worst }}=($ float $*) \operatorname{calloc}\left(N_{-}\right.$SIM, sizeof $($float $\left.)\right)$;

190:

191: // kernel para geracao dos caminhos aleatorios

192: generateRandomPath(h_Paths, h_Finals, h_Worst, h_tp, h_s0, K, h_vol, H, h_L);

193:

194: // Resultado final

195: float sum $=0$;

196: for (int $i=0 ; i<N_{-}$SIM; $i++$ ) \{

197: $\quad$ sum $+=$ h_Worst $[i]$;

198: \}

199: clock_t end $=\operatorname{clock}()$;

200: float time_elapsed = (float) (end - begin) / CLOCKS_PER_SEC;

201: printf("\nOption value: \% $\backslash \mathrm{n} "$, (sum / N_SIM)*exp $(-r * T)$ );

202: printf("Total time elapsed: \%f $s \backslash n "$, time_elapsed);

203:

204: return 0 ;

205:

206: \} 


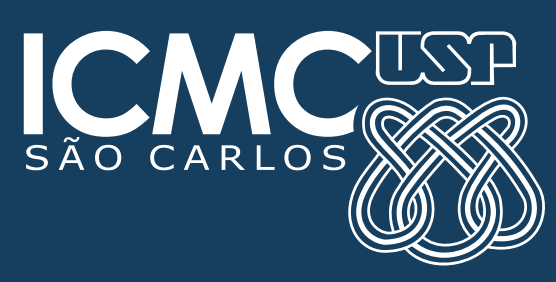

